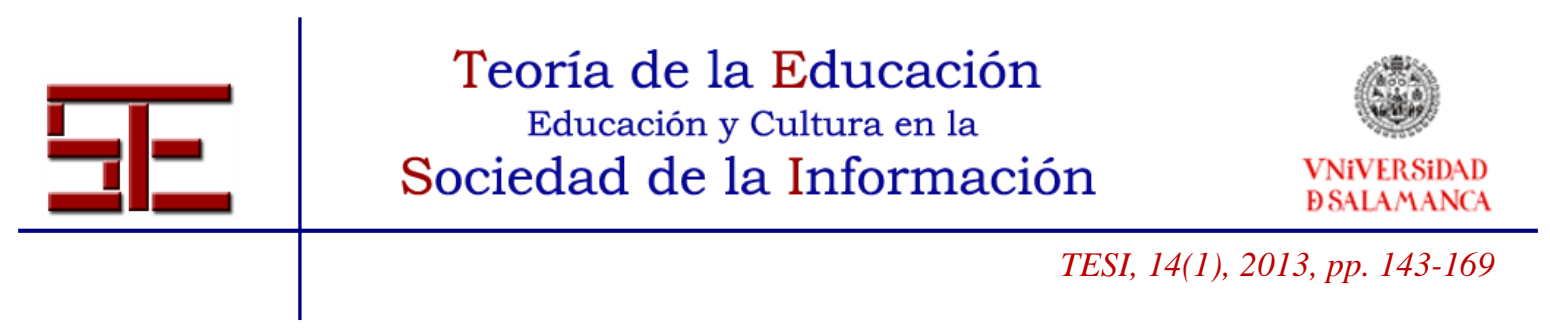

\title{
DISEÑO FUNCIONAL Y PROPUESTA DE IMPLEMENTACIÓN DE UNA HERRAMIENTA DE APOYO A LA CONSTRUCCIÓN COLABORATIVA DE CONOCIMIENTO
}

Resumen: Este artículo describe el proceso de conceptualización y diseño funcional de un foro electrónico, que se propone facilitar aquellos procesos de aprendizaje que toman como base la comunicación, pero también la colaboración y la construcción de conocimiento, así como su seguimiento y evaluación.

La experiencia se basa en un proyecto de innovación que implicó el diseño, el desarrollo y la implementación piloto de esta herramienta de comunicación asíncrona, en el contexto del campus virtual de la Universitat Oberta de Catalunya (UOC).

El diseño de la herramienta parte del análisis de distintos modelos de referencia en lo que respecta al apoyo tecnológico de procesos de aprendizaje colaborativo y de construcción social de conocimiento.

En paralelo, y tras analizar algunos modelos para el estudio de la construcción colaborativa de conocimiento en entornos virtuales, se presenta una propuesta de análisis y evaluación de este tipo de procesos mediados por herramientas de comunicación asíncrona similares a la diseñada.

Palabras clave: Aprendizaje colaborativo; construcción colaborativa de conocimiento; análisis de procesos de construcción colaborativa de conocimiento; herramientas de comunicación asíncrona; diseño de entornos de aprendizaje; foros de discusión.

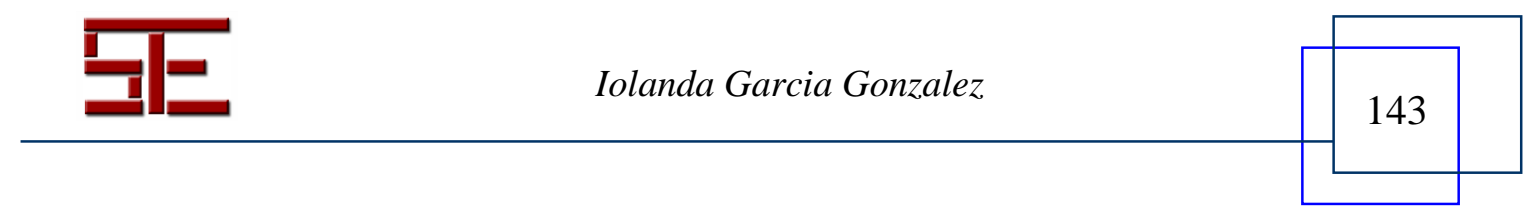




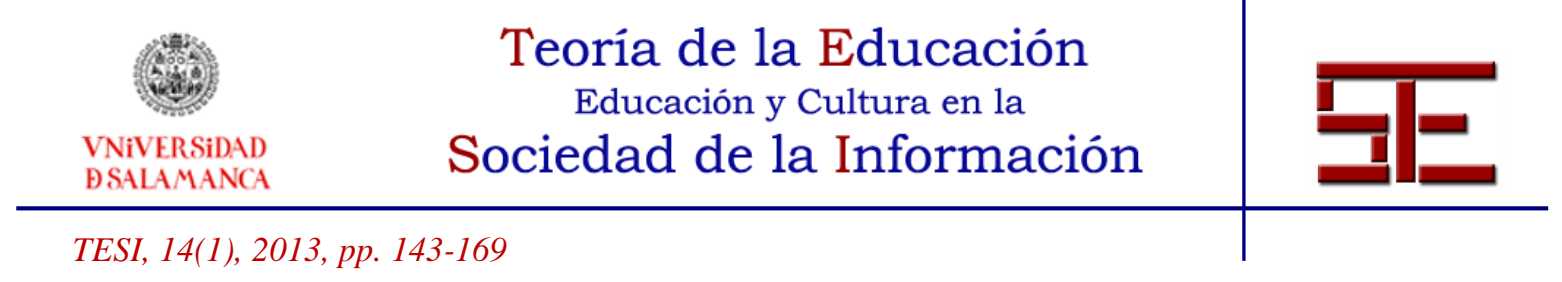

\title{
FUNCTIONAL DESIGN AND IMPLEMENTATION PROPOSAL OF A TOOL TO SUPPORT COLLABORATIVE KNWOLEDGE BUILDING
}

\begin{abstract}
This article describes the process of conceptualization and functional design of an electronic forum, which aims to facilitate learning processes based on communication, but also on collaboration and social knowledge building, and their monitoring and evaluation.
\end{abstract}

The experience is based on an innovation project involving the design, development and pilot implementation of an asynchronous communication tool, in the context of the Open University of Catalonia (UOC) virtual campus.

The design of the tool is based on the analysis of different reference models regarding the use of technology to support collaborative learning and social knowledge building processes.

In parallel, and after analyzing some models for the study of collaborative knowledge building processes in virtual environments, the article presents a proposal for the analysis and assessment of such processes mediated by asynchronous communication tools similar to the one designed.

Keywords: Collaborative learning; collaborative knowledge building; analysis of collaborative knowledge building processes; asynchronous communication tolos; design of learning environments; discussion forums.

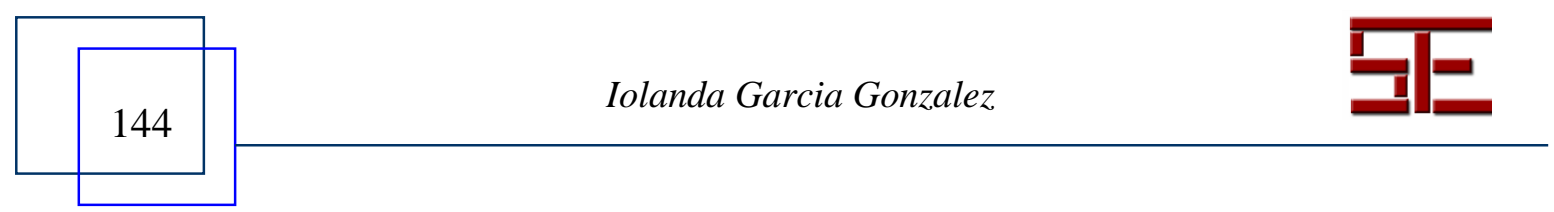




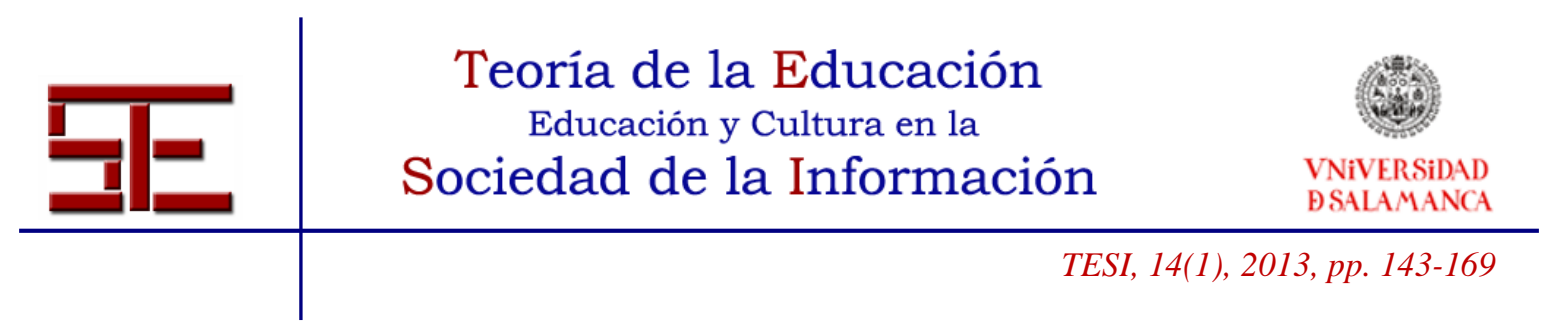

\section{DISEÑO FUNCIONAL Y PROPUESTA DE IMPLEMENTACIÓN DE UNA HERRAMIENTA DE APOYO A LA CONSTRUCCIÓN COLABORATIVA DE CONOCIMIENTO}

Fecha de recepción: 11/12/2012; fecha de aceptación: 04/02/2013; fecha de publicación: 28/02/2013

Iolanda Garcia Gonzalez

igarciago@uoc.edu

Universitat Oberta de Catalunya

\section{1.- INTRODUCCIÓN}

Si entendemos el aprendizaje como un proceso que tiene lugar en un contexto social compartido, de confrontación de significados, de negociación y de construcción conjunta, hemos de considerar a la comunicación como una dimensión fundamental y determinante de la calidad del aprendizaje.

En los últimos años ha tenido lugar un gran incremento del uso de entornos virtuales - o mediados por tecnología - en distintos contextos de formación. En la literatura se ha reconocido ampliamente el papel de las TIC como mediadoras y facilitadoras de los procesos de enseñanza y aprendizaje. Concretamente, los procesos comunicativos que tienen lugar en los entornos de aprendizaje virtual han sido objeto de numerosas investigaciones desarrolladas desde distintas perspectivas. A menudo, el análisis de la interacción se ha enmarcado en situaciones de colaboración y de construcción conjunta de conocimiento entre los estudiantes. No porque la comunicación social deba implicar sistemáticamente procesos de colaboración, sino por la popularidad que han ido ganando las metodologías colaborativas en los contextos de formación virtual y por el interés que ha despertado en particular la observación de los procesos interactivos dentro de estas dinámicas. En el campo de estudio de CSCL (Computer Supported Collaborative Learning), por ejemplo, dedicado a la investigación en torno al diseño y el uso de entornos tecnológicos de apoyo al aprendizaje colaborativo, se han analizado múltiples aspectos relacionados con la interacción colaborativa.

Como resultado de estas investigaciones, se ha puesto de manifiesto la dificultad que representa la generación de interacciones sociales de calidad, basadas en la reflexión y la argumentación fundamentada, que conduzcan hacia el intercambio de ideas y la construcción colaborativa de conocimiento. Esto convierte a los procesos de construcción colaborativa de conocimiento en entornos virtuales en un ideal al que aproximarse en

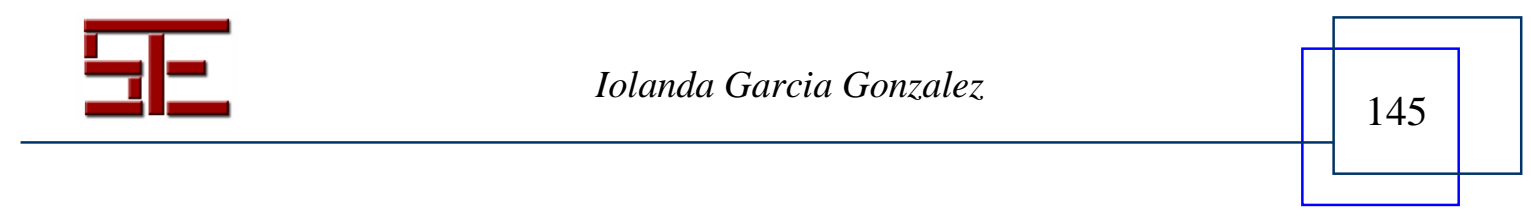




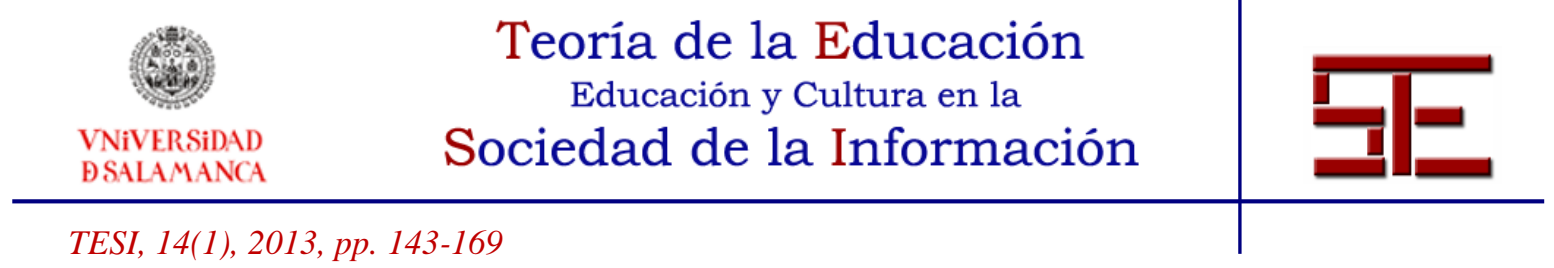

ciclos de entrenamiento y de interacción sucesivos, más que en una realidad que pueda plantearse como directamente asumible, de forma plena y durante un periodo de tiempo continuado.

A pesar del potencial formativo innegable de múltiples herramientas de apoyo a la comunicación y la colaboración disponibles en la actualidad, muchas experiencias de innovación e investigación encuentran a menudo dificultades en el momento de integrarlas a situaciones específicas de aprendizaje. Con frecuencia estas dificultades tienen que ver con un inadecuado diseño pedagógico de las actividades de aprendizaje. Sin embargo, existe otro factor a tener en cuenta que tiene que ver con el apoyo tecnológico. Y es que muchas herramientas de comunicación ya clásicas como los foros, y también otras aplicaciones mucho más nuevas de la web social, presentan deficiencias en su papel de facilitadoras de procesos directamente implicados en la colaboración, como la gestión y la organización de las intervenciones, la elaboración del conocimiento generado para su reutilización, el seguimiento ágil y eficaz de los procesos interactivos y de producción de discurso, o la evaluación de las intervenciones y del conocimiento que se va generando.

El amplio desarrollo del trabajo en el campo del CSCL parte de una constatación de la necesidad de mejorar las herramientas tecnológicas disponibles, con el fin de optimizar su papel de apoyo en los procesos interactivos que tienen lugar en contextos de formación en línea. Nos referimos concretamente a herramientas que puedan facilitar los procesos comunicativos, pero, más allá de ellos, también la colaboración y la producción de conocimiento.

Los espacios de discusión asíncrona y concretamente los foros y su papel en los procesos de formación han sido ampliamente estudiados desde distintas perspectivas, obteniéndose resultados diversos en función de factores como el tipo de soporte tecnológico utilizado, el diseño de las tareas, los contenidos trabajados, o incluso el diseño de las investigaciones y el enfoque teórico adoptado (Hammond, 2005). Sin embargo, lo que parecen indicar muchos de estos trabajos es que, a pesar de tratarse de actividades habituales y herramientas bastante generalizadas, que en principio ofrecen múltiples oportunidades en términos de aprendizaje colaborativo, el discurso que se genera en ellas como resultado no llega a reflejar, por lo general, una implicación cognitiva de alto nivel de los participantes (Coll, Bustos y Engel, 2011; Gunawardena, Lowe y Anderson, 1997; Lipponen, Rahikainen, Lallimo y Hakkarainen, 2003; Gros, 2004). Los estudios realizados coinciden en mostrar que, a menudo, no se establecen suficientes conexiones entre las distintas aportaciones. En general, se trata más bien de sucesiones de

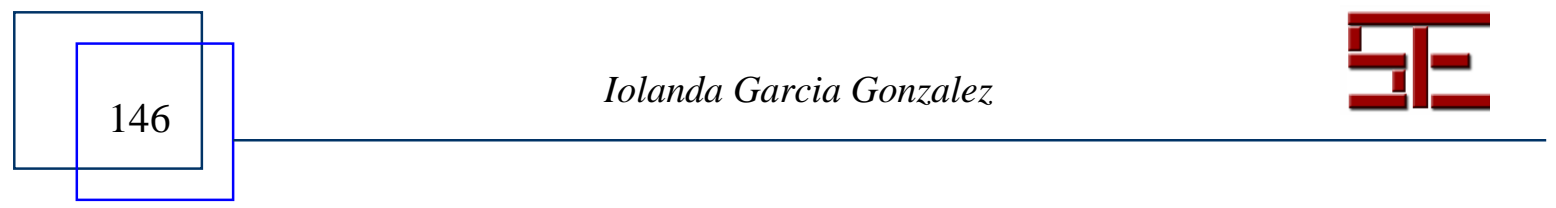




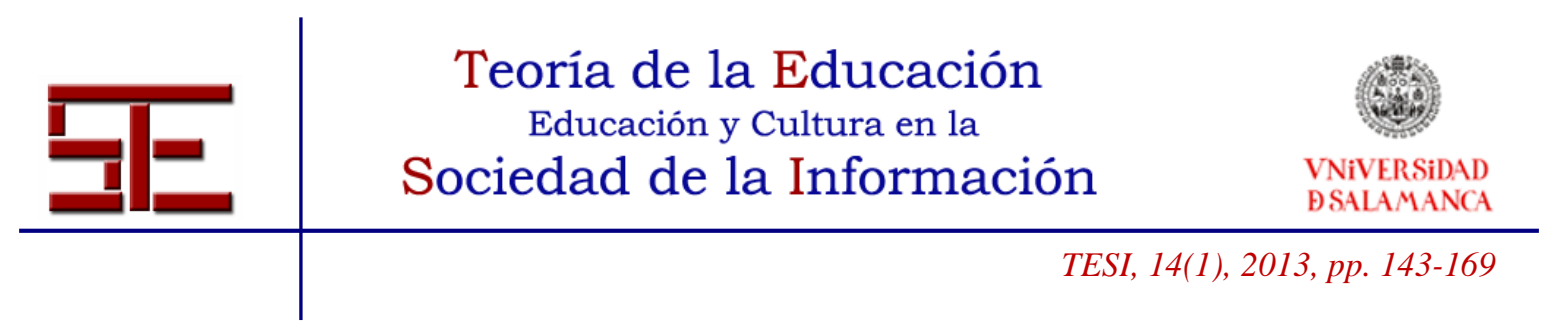

exposiciones condensadas de las propias ideas, sin que se preste demasiada atención a las ideas expuestas anteriormente por otros (Gao, 2011). A esta valoración, se añaden a menudo las dificultades para la dinamización y la moderación eficiente de las discusiones por parte de docentes y facilitadores. Si la discusión no se sostiene el tiempo suficiente es muy difícil que se generen los necesarios procesos de negociación de significados y de co-construcción a partir de las ideas aportadas (Gao, 2011; Guzdial y Turns, 2000; Hewitt, 2005 y Hew; Cheung, 2008).

Este artículo se basa en un proyecto de innovación que implicó el diseño, el desarrollo y la implementación piloto de una herramienta de comunicación asíncrona, en el contexto del Campus Virtual de la Universitat Oberta de Catalunya (UOC). En concreto, se describe y fundamenta la conceptualización y el diseño de un foro electrónico que se propone facilitar aquellos procesos de aprendizaje que toman como base la comunicación, pero también la colaboración y la construcción de conocimiento, así como su seguimiento y evaluación.

En paralelo, y tras analizar algunos modelos para el estudio de la construcción colaborativa de conocimiento en entornos virtuales, se presenta una propuesta de análisis y evaluación de este tipo de procesos mediados por herramientas de comunicación asíncrona similares a la diseñada.

\section{2.- CONTEXTO, OBJETIVOS Y FASES DEL PROYECTO}

El proyecto de innovación que presentamos ha sido financiado y desarrollado desde la Oficina Abierta de Innovación y el eLearn Center de la UOC con el título: "eKnowledge: desarrollo y pilotaje de una herramienta de foro para apoyar la construcción colaborativa de conocimiento en el Campus Virtual de la UOC".

El escenario de desarrollo del proyecto es la UOC, una universidad a distancia que gestiona toda la actividad formativa a través de un Campus Virtual, con más de 35.000 estudiantes distribuidos en distintos lugares del mundo. El foro actual a disposición general en el Campus Virtual es una herramienta de uso polivalente, utilizada tanto en los espacios de gestión como en las aulas, es decir, con fines formativos. En estas últimas funciona simultáneamente como espacio de comunicación informal en el que resolver dudas e intercambiar comentarios y a la vez como entorno para el desarrollo de debates de contenido curricular, más dirigidos. A pesar de haber ido incorporando algunas funcionalidades con el fin de mejorar la organización de las intervenciones, su estructura

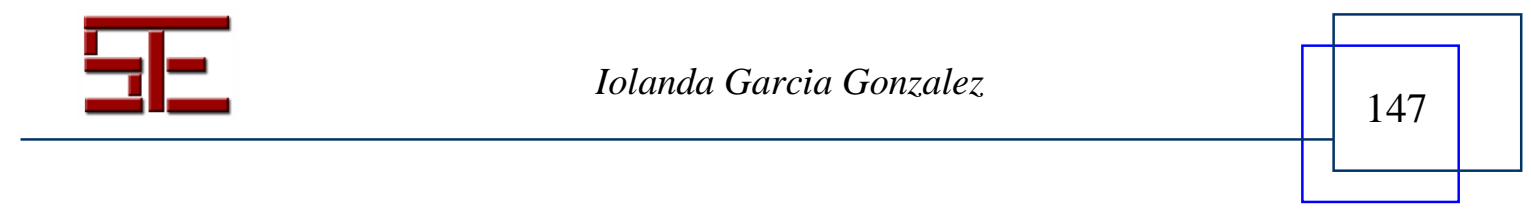




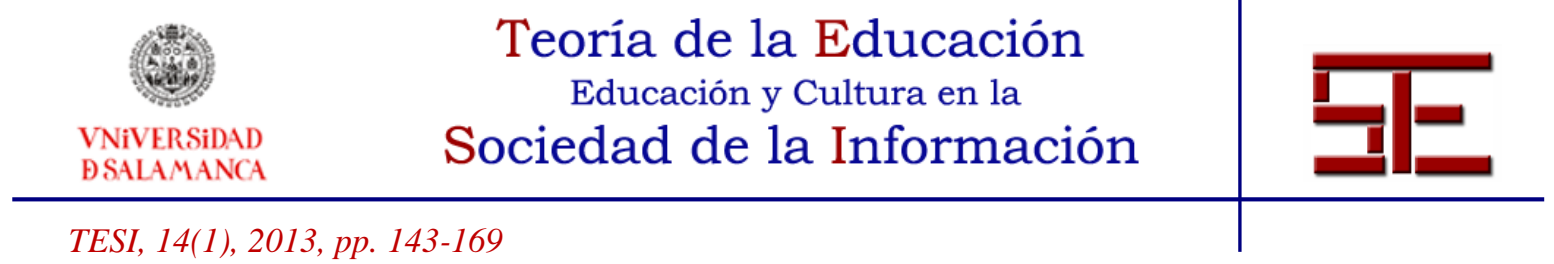

de base es poco flexible y no cuenta con algunas de las prestaciones ya comunes en los foros más actuales.

El modelo educativo de la UOC reconoce el componente social, inspirado en el concepto de presencial social planteado por Garrison, Anderson y Archer (2000), como esencial en el proceso de aprendizaje. La universidad reivindica de hecho, como uno de los valores importantes de su modelo formativo, el desarrollo de la competencia para el trabajo en equipo y en red entre sus estudiantes. Este enfoque pretende ser coherente con la filosofía de la web social o web 2.0, basada en la participación y en la construcción colectiva de conocimiento, desde planteamientos interdisciplinares, más transversales a la experiencia vital de los estudiantes (tanto formativa, como social y laboral). De hecho, los estudiantes utilizan ya herramientas 2.0 fuera del Campus Virtual por iniciativa propia, siendo de esperar que esta tendencia se incremente progresivamente.

Otorgar un papel fundamental a los procesos comunicativos y de trabajo conjunto entre los estudiantes implica la adopción de metodologías colaborativas en el diseño de las prácticas de aprendizaje que se propondrán a los estudiantes (AA.VV., 2009). Se trata de plantear situaciones de aprendizaje que exijan a los aprendices la coordinación de acciones conjuntas, la gestión compartida de la información y los recursos, la discusión y la argumentación de las propias ideas, la realización de juicios críticos sobre el trabajo de los compañeros, etc.

Si en el pasado fueron los materiales instruccionales y más tarde el aula virtual el foco de atención de los modelos de e-learning, los actuales se centran en garantizar la máxima flexibilidad y participación activa de los estudiantes en su experiencia de aprendizaje. Del mismo modo que la interacción social ha pasado a formar parte integral de la mayor parte de recursos formativos, las herramientas de comunicación pueden incorporar prestaciones de apoyo a procesos más complejos. Tal como mantienen Garrison y Anderson (2005), la presencia social interacciona de manera positiva con la presencia cognitiva, promoviéndola y manteniéndola, entendida esta última como procesos de pensamiento de alto nivel, condición para el logro de aprendizajes profundos (Kanuka y Garrison, 2004). Es decir, de alguna manera la frontera entre los usos estrictamente comunicativos y los más directamente asociados a la construcción de conocimiento tienden a desdibujarse, para pasar a entenderse como necesariamente complementarios.

A todo lo dicho hasta ahora, debemos añadir que la UOC trabaja con un modelo de evaluación continua desde hace ya unos años, que recientemente se ha visto reforzado por

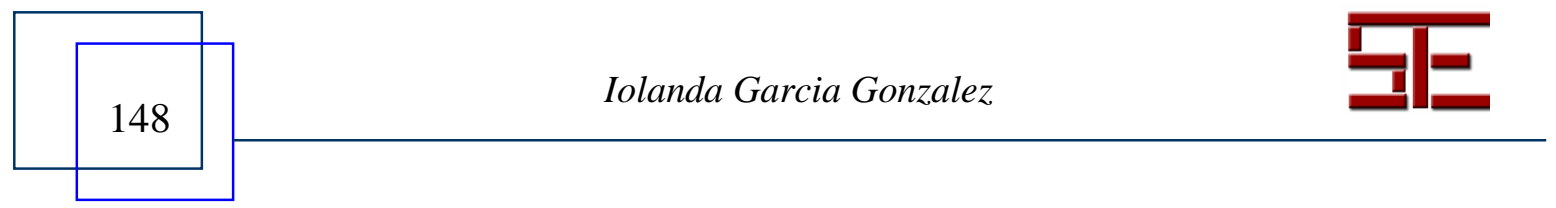




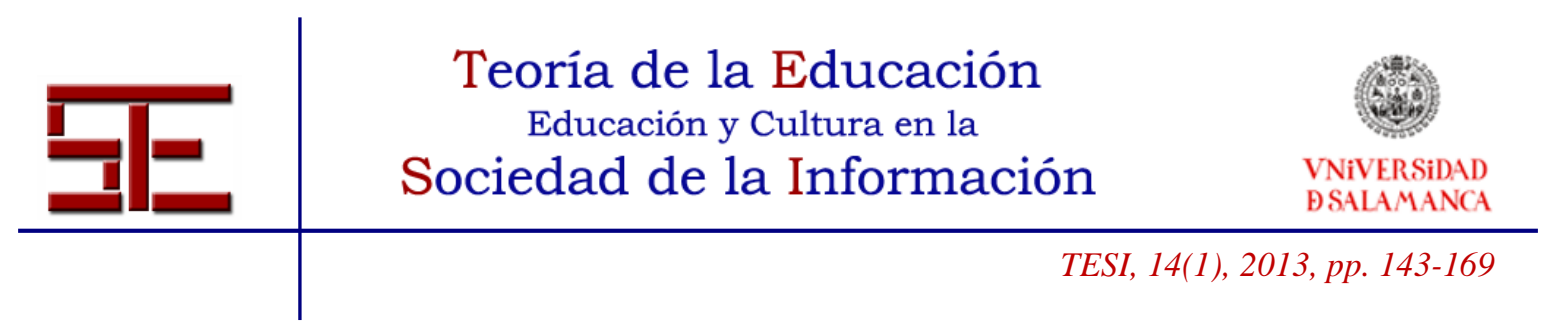

los planteamientos del Plan de Bolonia. En este marco, el papel de acompañamiento y seguimiento docente adquiere una importancia crucial.

Estas circunstancias del contexto actual hacen imprescindible pensar en sistemas de apoyo que favorezcan una gestión de la información más eficaz y faciliten el desarrollo de procesos comunicativos y colaborativos. Éstos deben favorecer la actividad interactiva de los estudiantes como sujetos de aprendizaje, pero también la del docente, como modelador y guía de los procesos de aprendizaje conjunto. Esto nos plantea la necesidad de pensar en instrumentos que faciliten la estructuración y el seguimiento por parte del profesor. Es decir, herramientas que faciliten la gestión de las prácticas de enseñanza, por una parte, y la (auto)gestión de los procesos de aprendizaje, por otra.

En concreto, los objetivos del proyecto se proponen dar respuesta a las necesidades de la institución a dos niveles distintos:

1. Diseñar una herramienta de comunicación asíncrona que responda al amplio repertorio de situaciones interactivas que tienen lugar en la institución.

2. Incorporar en el diseño de la herramienta prestaciones que faciliten su integración en modelos metodológicos basados en el aprendizaje colaborativo y la construcción de conocimiento.

El primer objetivo plantea por lo tanto la necesidad de mejorar las funcionalidades de navegación y de gestión de la información y el segundo objetivo requiere de la integración de funcionalidades expresamente concebidas para favorecer interacciones que conduzcan a la reflexión, la negociación, la argumentación conjunta, y en definitiva la producción colaborativa de conocimiento.

La Figura 1 muestra las fases principales de desarrollo del proyecto. Éste se inicia el curso 2008-2009, en el que se trabaja en colaboración con una empresa dedicada al desarrollo de proyectos online en la conceptualitzación de la herramienta y el diseño del primer prototipo. En la segunda fase se realiza una primera evaluación de la maqueta diseñada, por parte de dos colectivos distintos: un grupo de docentes de entornos de formación en línea y un conjunto de usuarios de los foros online del portal web emagister.com.

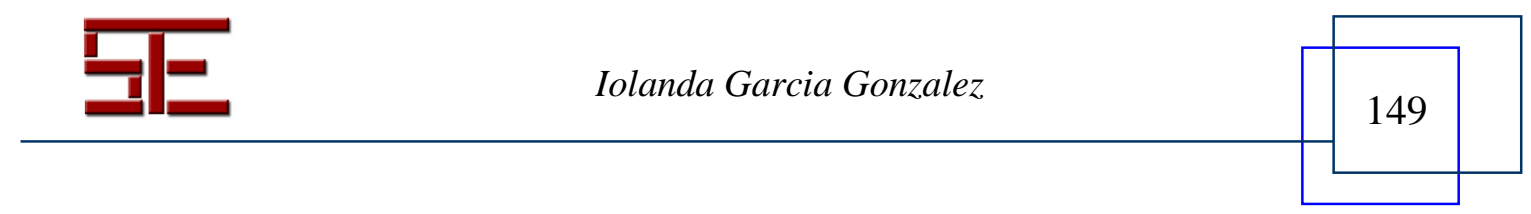



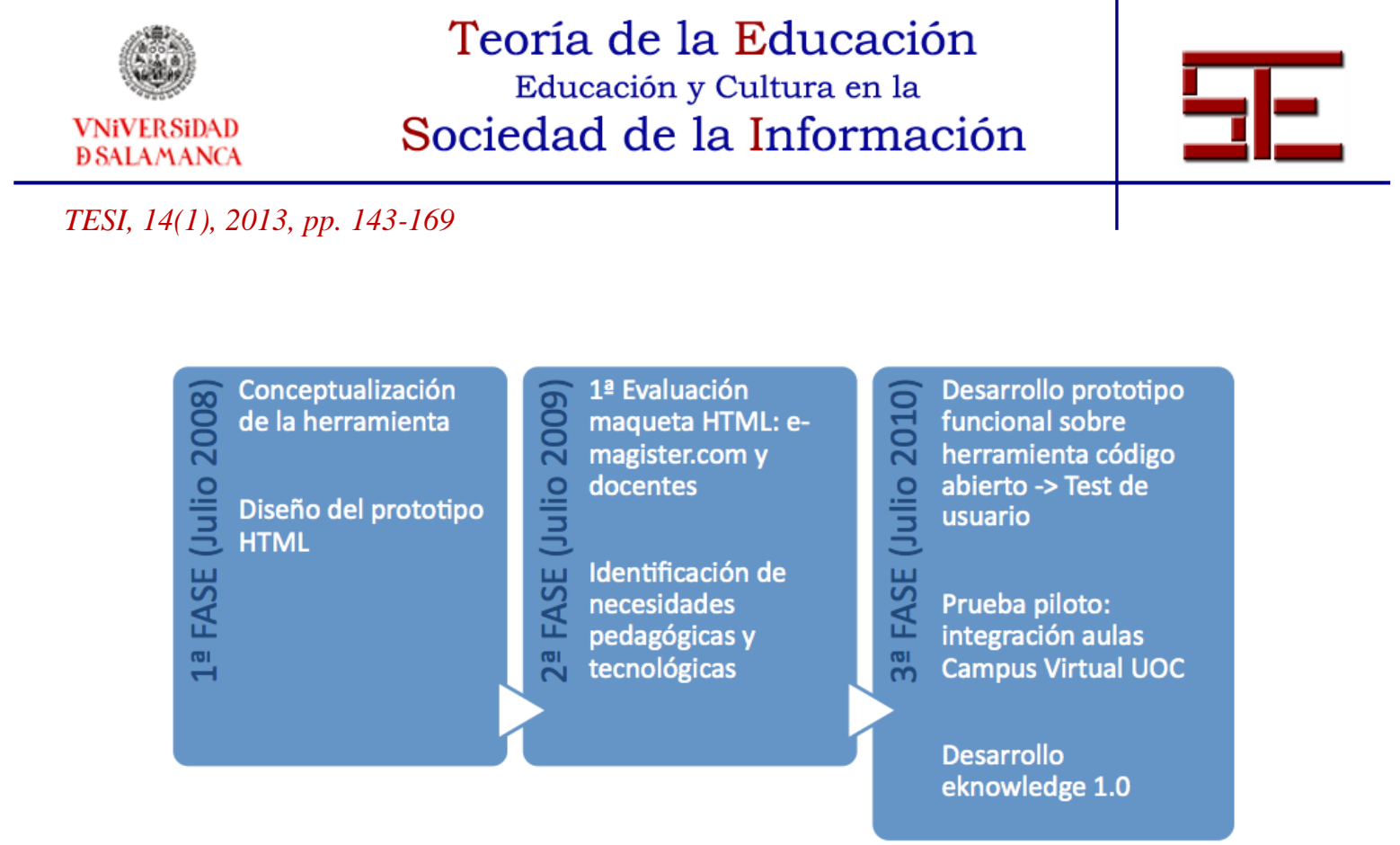

Figura 1. Fases de desarrollo del proyecto

Durante el curso 2009-2010 se desarrolla la tercera fase, en la que se trabaja conjuntamente con el Área de Tecnología Educativa de la universidad. En esta etapa tiene lugar la adaptación del diseño y el desarrollo de la herramienta y se constituye un grupo integrado por 8 profesores de diferentes estudios (Ciencias de la Información y de la Comunicación, Informática, Multimedia y Telecomunicación, Economía y Empresa y Psicología y Ciencias de la Educación) interesados en el uso formativo de herramientas de foro. El papel de este grupo es, por una parte, el de contribuir y validar el diseño definitivo de la herramienta $y$, por otra, preparar una prueba piloto en aquellas asignaturas que presenten las condiciones adecuadas. Así, a lo largo de este curso tienen lugar una serie de reuniones en las que se presenta la maqueta disponible y se discute el diseño de la misma, tanto desde el punto de vista pedagógico como tecnológico. Finalmente, el primer semestre del curso 2010-2011 se desarrollan las primeras pruebas piloto de la herramienta en las que participan consultores y estudiantes de varias asignaturas de los estudios referidos. En conjunto, se calcula que toman parte en estas pruebas más de 200 estudiantes, así como 11 consultores distribuidos en 11 aulas virtuales.

En los dos apartados siguientes realizamos una síntesis del trabajo desarrollado en la fase de conceptualización y describimos los rasgos principales del diseño resultante.

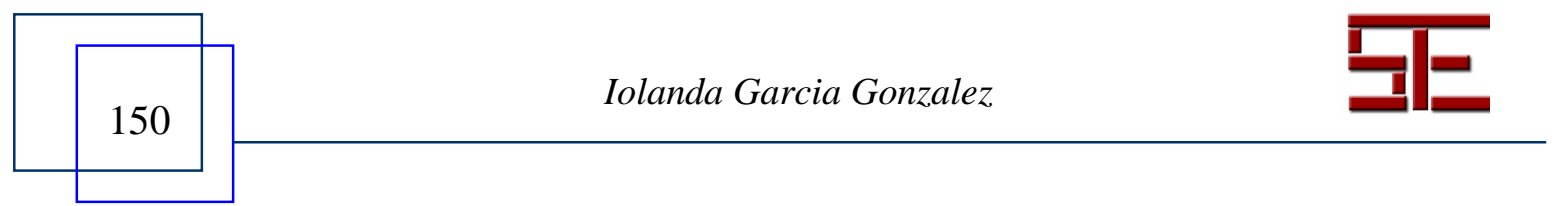




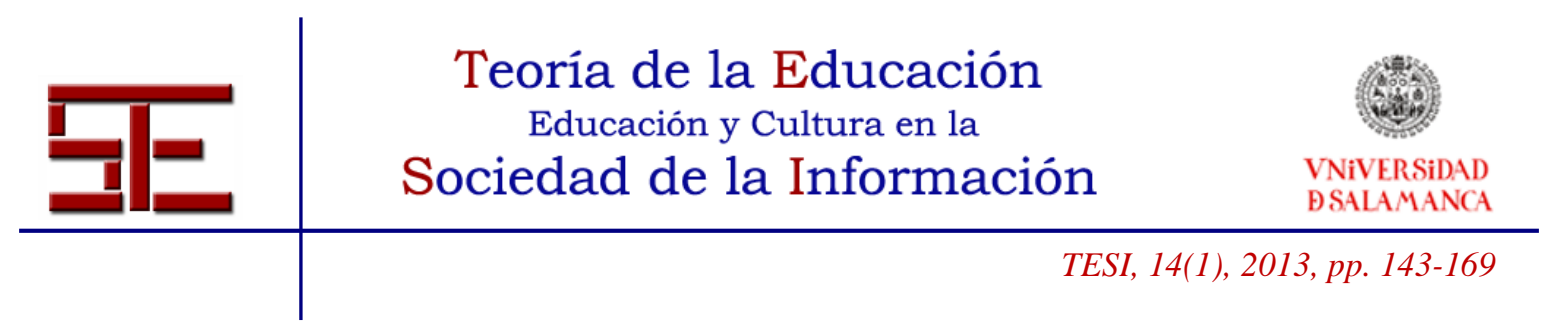

\section{3.- FUNDAMENTACIÓN DEL DISEÑO DE UNA HERRAMIENTA DE APOYO A LA CONSTRUCCIÓN COLABORATIVA DE CONOCIMIENTO}

En la fase de conceptualización inicial se identificaron y definieron los requerimientos de los dos niveles de prestaciones siguientes:

NIVEL 1: Relacionado con la usabilidad de la herramienta, centrado en la mejora de las posibilidades de navegabilidad y de gestión de la información.

NIVEL 2: Centrado en las funcionalidades de apoyo al proceso o la metodología de trabajo y de aprendizaje, orientado concretamente a la mediación del aprendizaje colaborativo y la construcción de conocimiento.

Ambos niveles de prestaciones se trabajaron de forma integrada, ya que varias funcionalidades debían ser abordadas desde ambos niveles. Más allá de su dimensión tecnológica, entendemos el concepto de prestaciones (affordances) de un entorno virtual en el sentido que son definidas por Kirschner, Strijbos, Kreijns y Beers (2004): en su dimensión social - como propiedades que actúan como facilitadoras socio-contextuales en los procesos de interacción social de los estudiantes (prestaciones sociales)- ; y en su dimensión educativa -como propiedades que determinan si una acción de aprendizaje específica podría producirse en un contexto determinado y cómo-.

Con el fin de concretar los rasgos y funcionalidades específicas que incorporar en cada nivel de prestaciones se analizan tres modelos de referencia en el diseño de herramientas de apoyo a la comunicación y la colaboración:

A. Programas diseñados en el campo del CSCL, con una fundamentación teórica específica (como por ejemplo Knowledge Forum; Future Learning Environment; Basic Support for Collaborative Learning, etc.).

B. Herramientas de discusión (foros electrónicos) de plataformas generalistas de elearning (como por ejemplo los foros de Moodle).

C. Herramientas enmarcadas en el enfoque de la web social o web 2.0 (como por ejemplo los foros phpBB, de Zotero, etc.).

En la Tabla 1 presentamos una síntesis del análisis realizado a partir de productos representativos de cada modelo de referencia, con la intención de valorar las ventajas y limitaciones de cada uno de ellos.

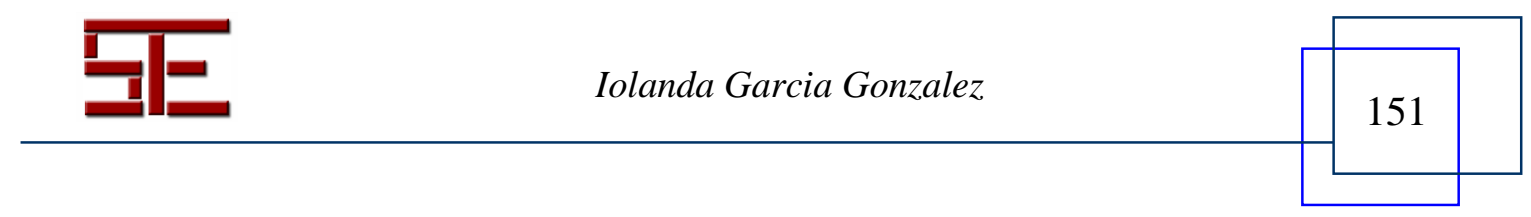




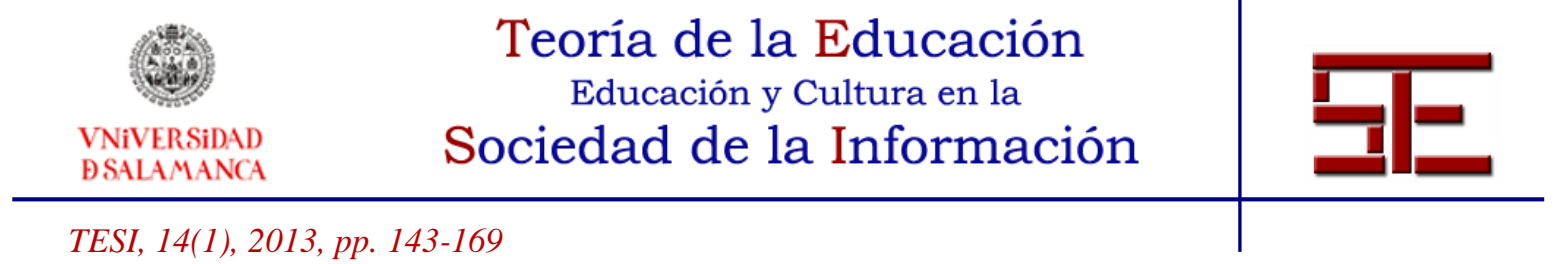

En el campo del diseño de sistemas de CSCL se ha dado un paso más allá en los últimos años, que ha permitido el desarrollo de entornos expresamente concebidos para asistir procesos argumentativos, de construcción social de conocimiento y de focalización de las discusiones online. Las investigaciones realizadas en este campo sugieren que el diseño de un entorno virtual puede afectar la naturaleza de las discusiones en línea, al influir en aquello que los participantes pueden ver y hacer. De este modo, mediante un diseño adecuado es posible también promover una característica o cualidad concreta en las discusiones en línea.

De acuerdo con Bereiter y Scardamalia (2003) la función de los entornos colaborativos es dotar de una estructura a la colaboración y actuar como mediadores de los procesos de reflexión y de construcción de conocimiento. Estos investigadores han desarrollado la teoría de la construcción colaborativa de conocimiento y son también los creadores del programa Knowledge Forum (Scardamalia y Bereiter, 2002), diseñado a partir de este modelo y concebido expresamente para apoyar los procesos discursivos derivados. El programa KF es un referente dentro del campo del CSCL y ha inspirado, desde su creación, el desarrollo de otras herramientas.

Otros dos ejemplos de herramientas fundamentadas en un modelo similar son Fle3 (Future Learning Environment) y BSCL (Basic Support for Collaborative Learning), ambas desarrolladas en el proyecto europeo ITCOLE. Este proyecto se propuso crear herramientas de apoyo a modelos colaborativos de aprendizaje, aplicables en el contexto escolar de una serie de países, buscando la adaptabilidad de dichos modelos y herramientas a diferentes contextos y necesidades.

En el primer caso, se trata de una herramienta basada en el modelo de cuestionamiento progresivo impulsado desde el Centre for Research on Networked Learning and Knowledge Building de la Universidad de Helsinki en Finlandia (Leinonen, Virtanen, Hakkarainen y Kligyte, 2002). La segunda se desarrolla a partir de la colaboración entre Gerry Stahl, de la Drexel University, y el Media Lab de Helsinky. En este caso se basa en la teoría de la cognición grupal elaborada por Stahl (2006). Las aportaciones de la teoría de Stahl al desarrollo del entorno consisten en la inclusión de funcionalidades que permitan manejar el discurso del grupo, y así favorecer una comprensión compartida y la negociación de nuevos significados entre sus miembros.

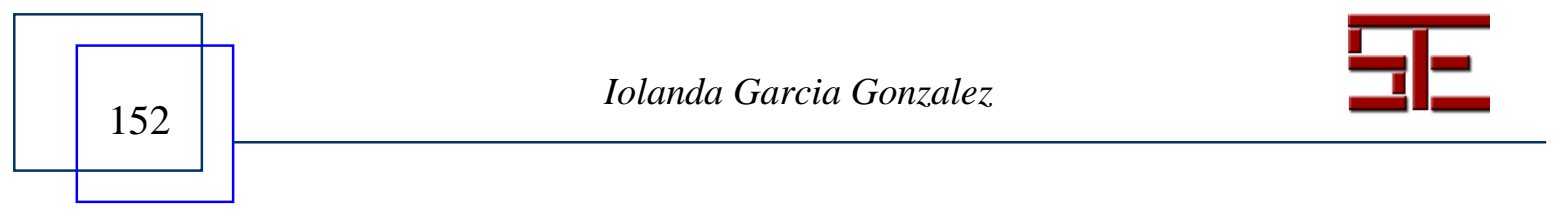




\begin{tabular}{c|c} 
Teoría de la Educación \\
Educación y Cultura en la \\
Sociedad de la Información
\end{tabular}

\begin{tabular}{|c|c|c|}
\hline \multicolumn{3}{|c|}{ MODELO 1: HERRAMIENTAS CAMPO CSCL } \\
\hline & APORTACIONES & LIMITACIONES \\
\hline 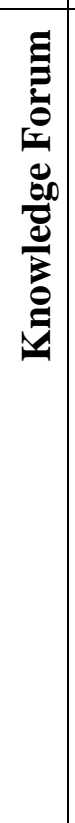 & 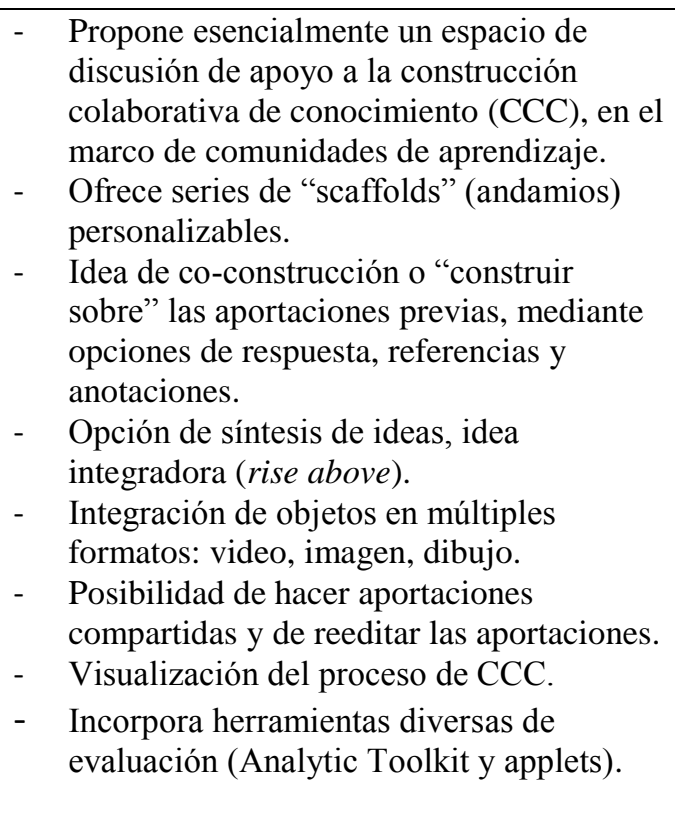 & 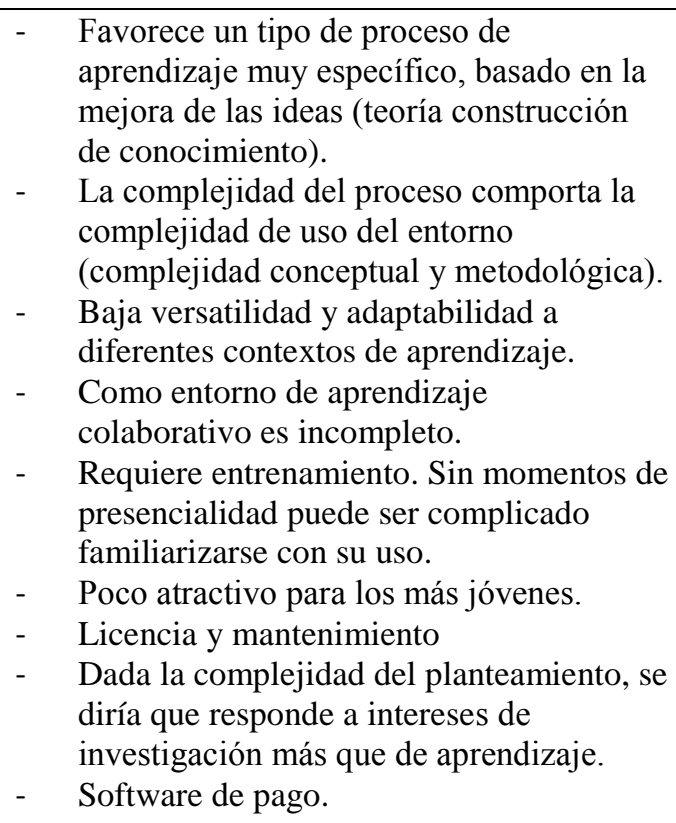 \\
\hline 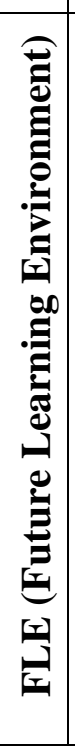 & 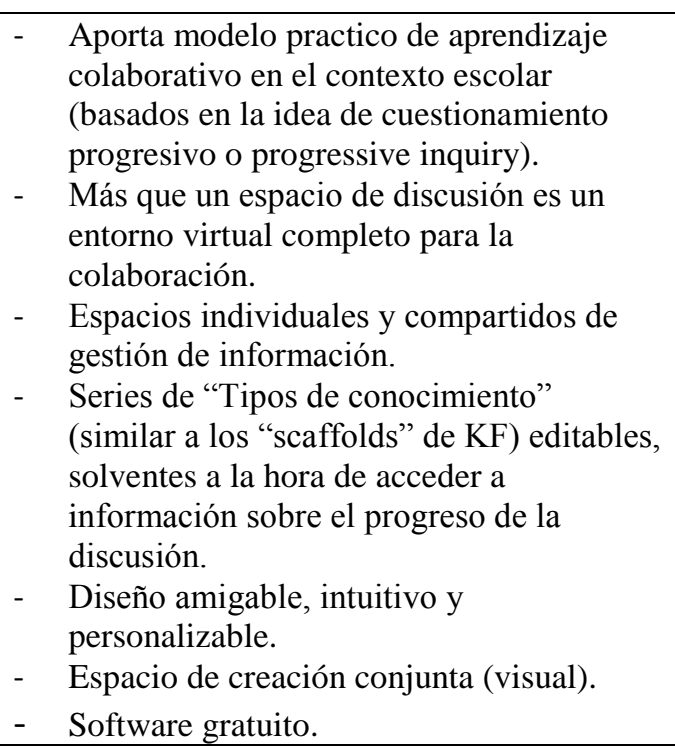 & 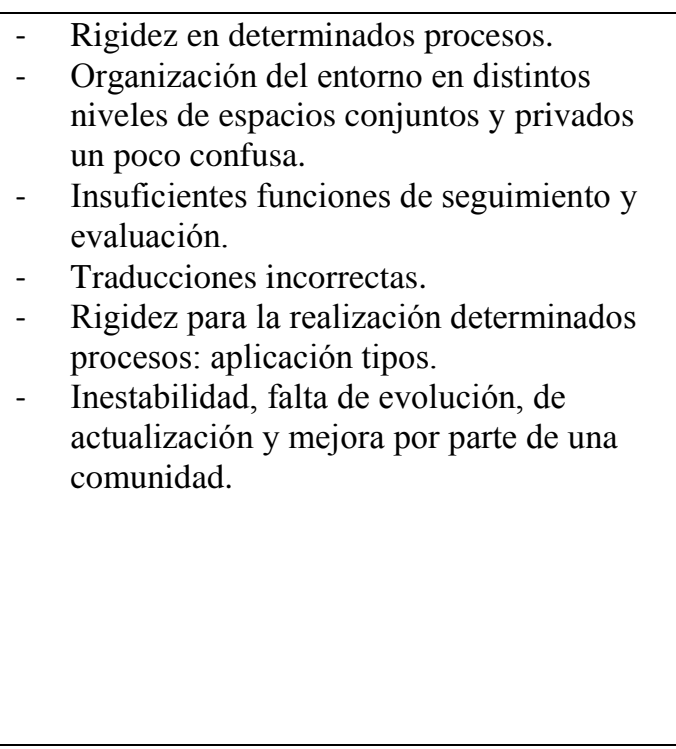 \\
\hline
\end{tabular}

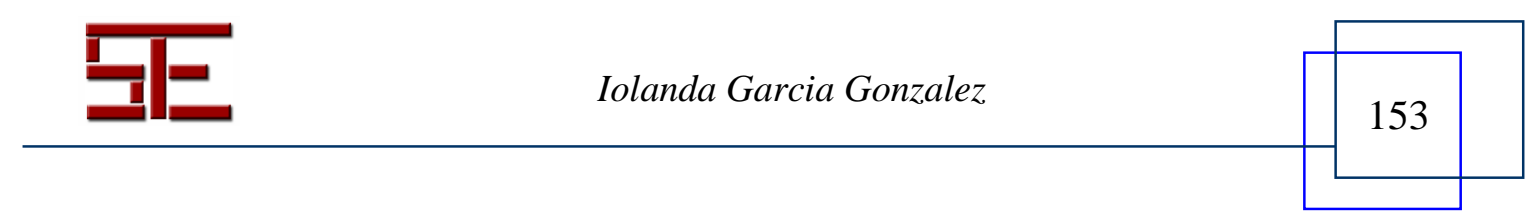




Teoria de la Educación
Educación y Cultura en la
$\begin{gathered}\text { VNiVERSIDAD } \\ \text { BSALAMANCA }\end{gathered}$

\begin{tabular}{|c|c|c|}
\hline 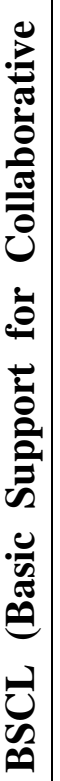 & 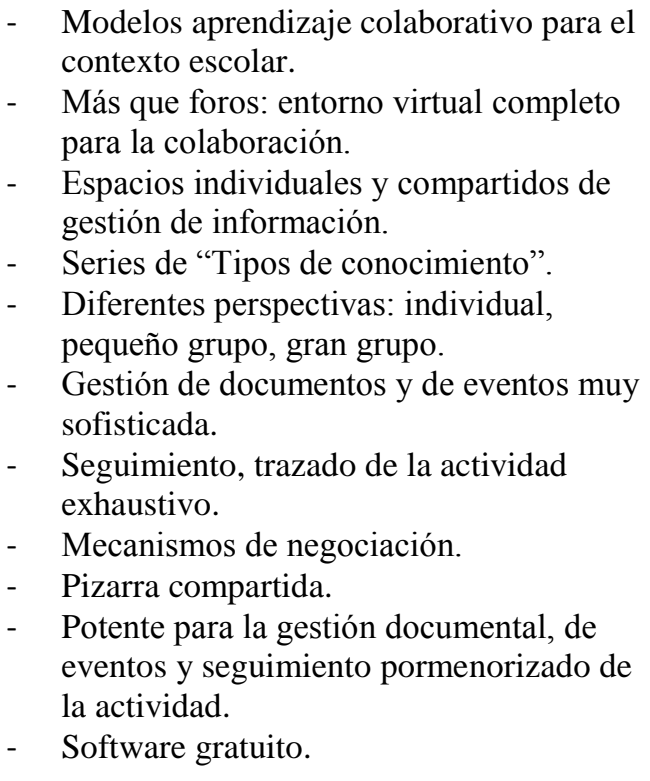 & $\begin{array}{ll}\text { - } & \text { Complejidad gestión de espacios y } \\
\text { participantes. } \\
\text { - }\end{array}$ \\
\hline
\end{tabular}

Tabla 1. Modelos de referencia en el diseño de herramientas comunicativas y colaborativas (1/2)

Los foros de las plataformas generalistas, cuyo diseño no se fundamenta de manera estricta en un modelo teórico, como podría ser el caso de Moodle, se configuran como espacios eminentemente comunicativos. En general, están controlados por un admistrador con privilegios especiales para su gestión, que suele ser el propio docente. Se basan en el intercambio de mensajes textuales, la adjunción de documentos y, en algunos casos, la integración de enlaces y elementos multimedia en el cuerpo del mensaje. Ciertos foros han empezado a incorporar la posibilidad de grabar mensajes sonoros. Por lo general, los mensajes se organizan en secuencias encadenadas que permiten reconocer el desarrollo del diálogo (Feliz y Ricoy, 2008). Estas cadenas o hilos de discusión tienen un papel importante en los contextos formativos puesto que, por una parte, conectan el discurso promoviendo una interacción más productiva entre los participantes $\mathrm{y}$, por otra parte, hacen visibles estas conexiones, cosa que facilita el seguimiento de las intervenciones.

Sin embargo, la investigación desarrollada en este ámbito muestra que la estructura en hilos de conversación puede no ser suficiente para conectar y sostener una discusión, ya que su estructura no siempre facilita la visualización de las conexiones y relaciones entre las aportaciones individuales. En la mayoría de foros, inclusive los desarrollados desde el enfoque de la web social, los mensajes se presentan en orden lineal y cronológico, y aunque se utilice una estructura en árbol para reflejar la jerarquía de las aportaciones,

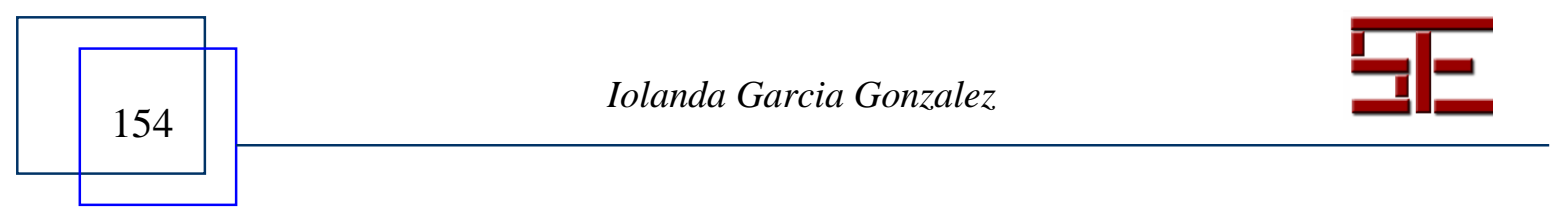




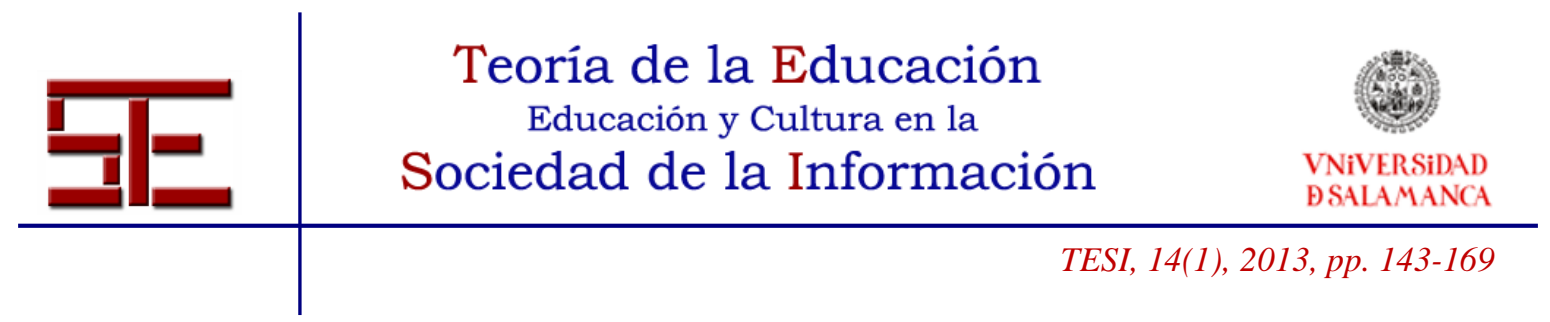

continúa siendo complicado visualizar cómo se interelacionan los mensajes entre sí. Estas estructuras dificultan así mismo la comprensión del desarrollo y mantenimiento de las discusiones, de modo que en una discusión con abundantes aportaciones, los participantes tienden a perder la perspectiva global de la discusión y a focalizarse en dar respuesta a mensajes particulares, sin atender a la organización en temas y subtemas. Esto termina dando lugar a discusiones con un bajo nivel de coherencia y profundidad (Thomas, 2002; Gao, 2011).

\begin{tabular}{|c|c|c|}
\hline \multicolumn{3}{|c|}{ MODELO 2. HERRAMIENTAS DE PLATAFORMAS DE E-LEARNING } \\
\hline & APORTACIONES & LIMITACIONES \\
\hline 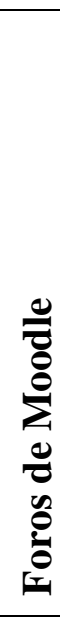 & $\begin{array}{ll} & \text { Tipos de foros predeterminados: diferentes } \\
\text { tipologías pensadas para estructurar distintos } \\
\text { tipos de procesos interactivos en situaciones } \\
\text { formativas. } \\
\text { - } \\
\text { - } \\
\text { Dstructuración de la discusión en temas opciones de organización y } \\
\text { visualización de la discusión. } \\
\text { - Posibilidad de asignación de roles/privilegios } \\
\text { a distintos tipos de participantes. } \\
\text { - Herramientas para el seguimiento de los } \\
\text { participantes. } \\
\text { - } \\
\text { Herramienta para la evaluación de los } \\
\text { participantes. } \\
\text { - } \quad \text { Re)edición de las aportaciones. } \\
\text { Software gratuito y open source. }\end{array}$ & $\begin{array}{ll}\text { - } & \text { Opciones limitadas en la edición de las } \\
\text { aportaciones y la inclusión de elementos } \\
\text { multimedia. }\end{array}$ \\
\hline \multicolumn{3}{|c|}{ MODELO 2. HERRAMIENTAS DE LA WEB SOCIAL O 2.0 } \\
\hline & APORTACIONES & LIMITACIONES \\
\hline
\end{tabular}

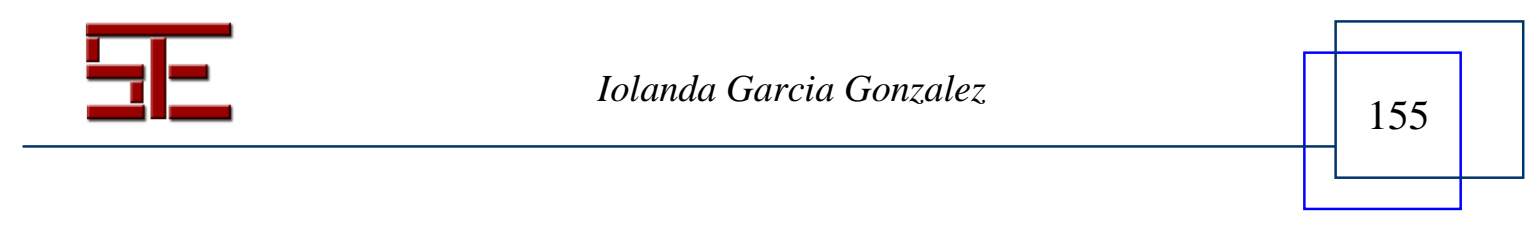




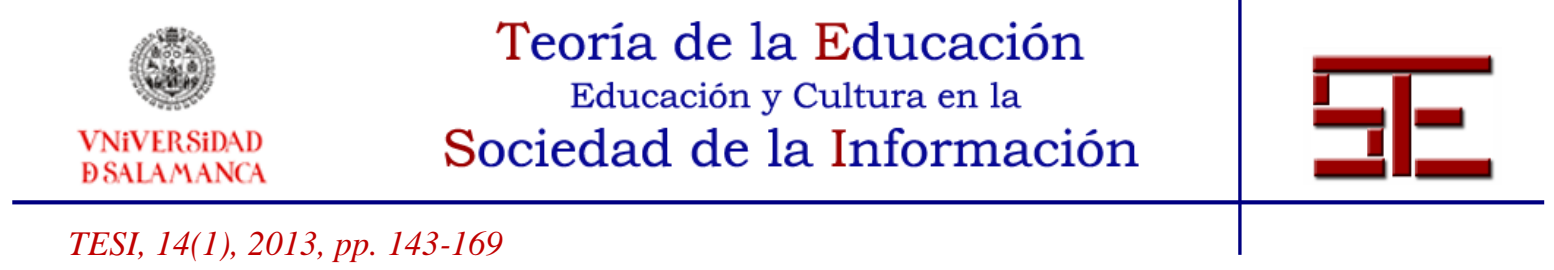

\begin{tabular}{|c|c|c|}
\hline 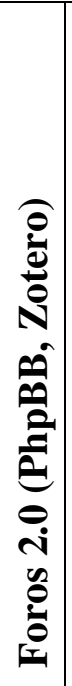 & 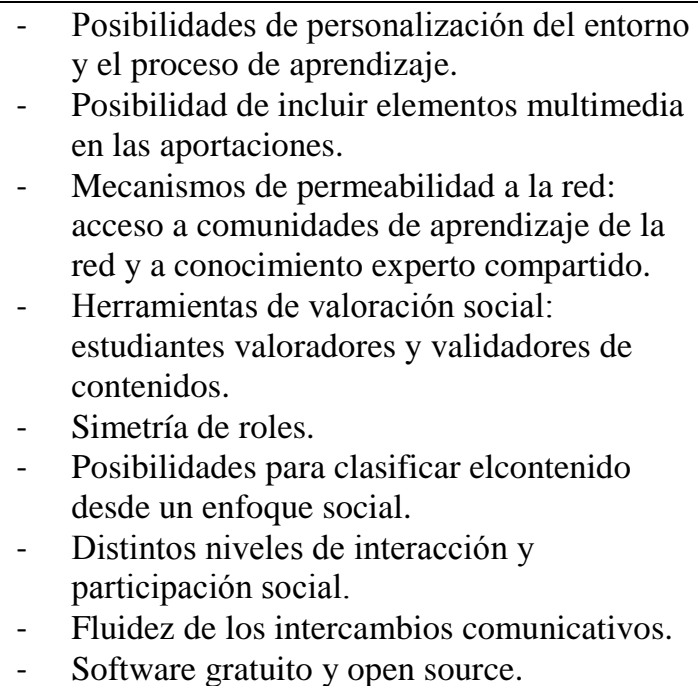 & $\begin{array}{l}\text { - } \quad \text { Falta de estructuración del proceso. La } \\
\text { estructuración prefijada va contra la } \\
\text { naturaleza de este tipo de herramientas. } \\
\text { - } \quad \begin{array}{l}\text { Dificultades para una gestión personal de } \\
\text { la información eficiente. }\end{array} \\
\text { - } \quad \begin{array}{l}\text { Dificultad seguimiento y evaluación } \\
\text { (trazabilidad). }\end{array} \\
\text { - } \quad \begin{array}{l}\text { Dificultad gestión y validación del } \\
\text { conocimiento generado. }\end{array} \\
\text { - La organización de la información } \\
\text { responde a los criterios de los estudiantes } \\
\text { y es de difícil control por parte del } \\
\text { docente. }\end{array}$ \\
\hline
\end{tabular}

Tabla 1. Modelos de referencia en el diseño de herramientas comunicativas y colaborativas (2/2)

Por último, las herramientas comunicativas de la web social comparten una visión de cualquier usuario como creador de contenidos que se refleja en la alternancia de roles. Esta característica unida a la propiedad compartida de las contribuciones dificulta en ocasiones el seguimiento de las mismas. Se basan en la interconexión de contenidos y micro-interacciones entre múltiples usuarios, incluso entre distintas plataformas, por lo que permiten, de forma mucho más eficiente que en los anteriores modelos, la integración de saberes desarrollados en distintos ámbitos (de aprendizaje formal, no formal, profesional, personal, social, etc.). En general, la participación en estos entornos permite abrirse a una comunidad más amplia que la del aula o el curso, a pesar de que también puede circunscribirse a éstas. Lo natural es que los criterios de calidad para el reconocimiento de las aportaciones pasen a ser sociales y compartidos, y no controlados por el docente o un experto (Lomas, Burke y Page, 2008). Estas características, entre otras, nos llevan a concluir que las herramientas y entornos aglutinados por este modelo de referencia resultan más solventes a la hora de vehicular el componente de presencia social al que nos referíamos antes. Según lo plantean Garrison, Anderson y Archer $(2000,94)$ este se define como "la capacidad de los participantes para proyectarse a sí mismos social y emocionalmente, como personas 'reales', en una comunidad de investigación, mediante los medios de comunicación en uso". Sin embargo, la estructura eminentemente abierta y descentralizada de estas herramientas hace difícil su uso en situaciones de aprendizaje más constreñidas a unos parámetros concretos. Por este motivo, su utilización y sobre todo el aprovechamiento pleno de sus posibilidades puede

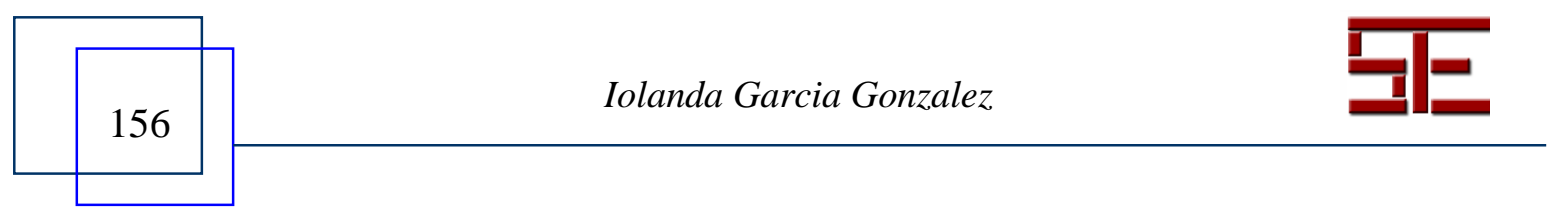




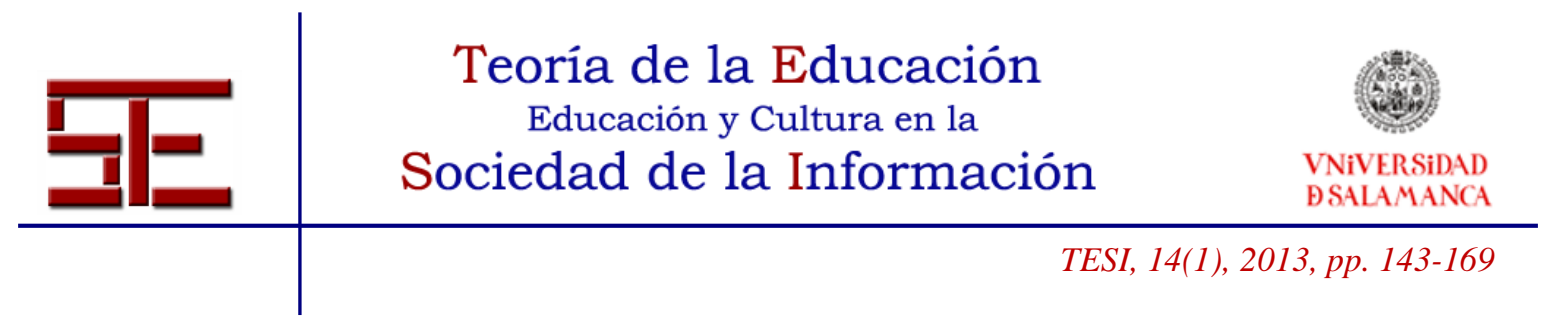

hacer necesaria la modificación del planteamiento habitual de las actividades de aprendizaje, así como de los sistemas de seguimiento y evaluación de las mismas.

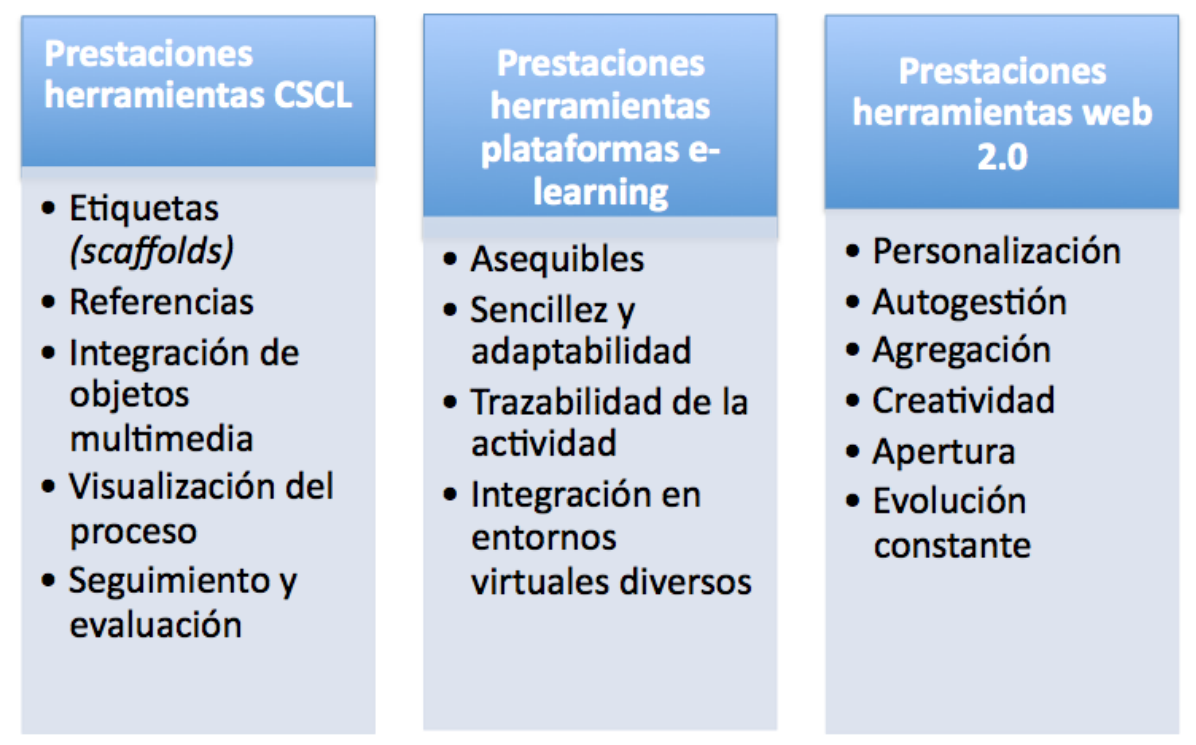

Figura 2. Resumen de características y funcionalidades relevantes en cada modelo de referencia

\section{4.- DISEÑO FUNCIONAL DE LA HERRAMIENTA eKNOWLEDGE}

Como resultado del análisis de los modelos de referencia mencionados, identificamos una serie de prestaciones relevantes para favorecer el tipo de procesos deseados, que aparecen resumidas en la Figura 2. Añadimos a continuación unos principios o requerimientos generales que nos parecen importantes para situar el enfoque adoptado en el diseño de eKnowledge:

a) Usabilidad: Debe favorecer procesos complejos pero sin renunciar a su propia simplicidad. Debe permitir una interacción natural, mediante una interfaz fácil de comprender y de usar.

b) Capacidad de apoyo al aprendizaje: Debe responder a requisitos de enseñanza y aprendizaje más que a intereses de investigación, o mero intercambio social. A la vez, debe ser versátil y adaptable a diferentes situaciones comunicativas y contextos de aprendizaje.

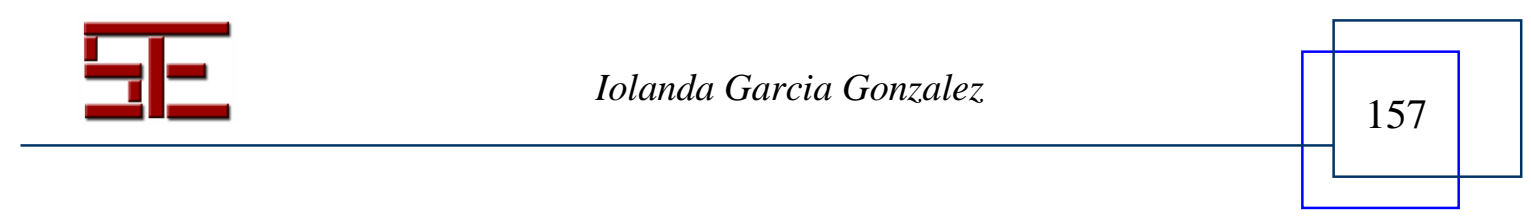




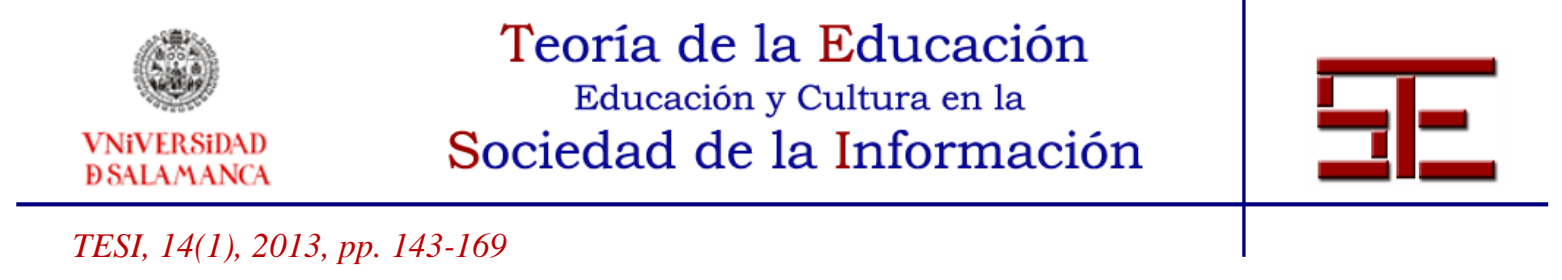

c) Motivación y personalización: Debe invitar al intercambio social y favorecer un diálogo sostenido. Debe ser configurable por parte del docente y, a la vez, personalizable por parte de cada estudiante.

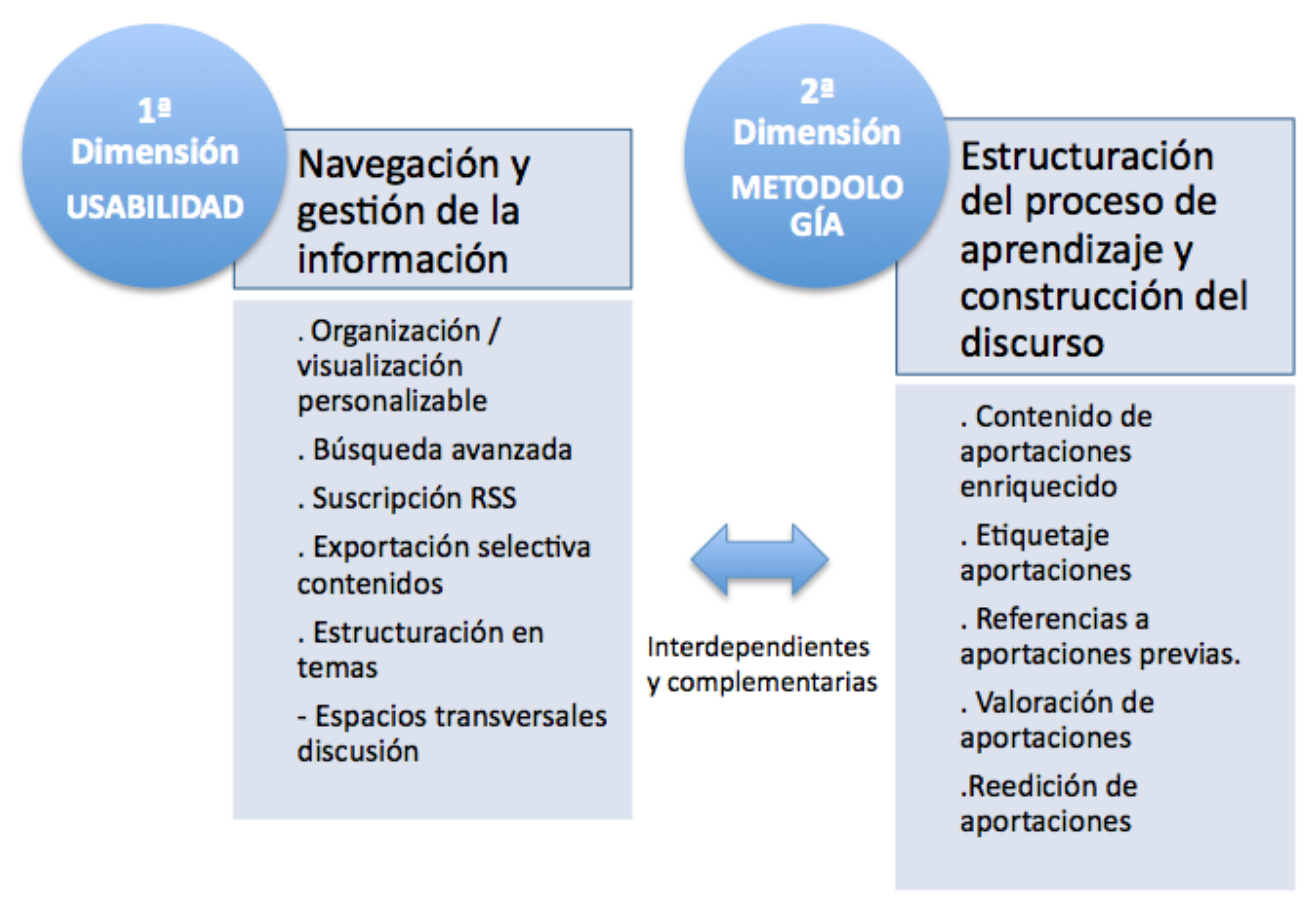

Figura 3. Estructura de prestaciones y funcionalidades de la $1^{\mathrm{a}}$ maqueta de eKnowledge

El resultado de la primera fase de conceptualización fue una maqueta funcional de la herramienta. Esta maqueta se utilizó como punto de partida para el desarrollo de un primer prototipo de la herramienta integrable al Campus de la UOC. En la Figura 3 se sintetizan algunas de las características y funcionalidades más importantes de la primera maqueta de la herramienta, siguiendo la clasificación de los dos bloques o dimensiones que indicábamos previamente.

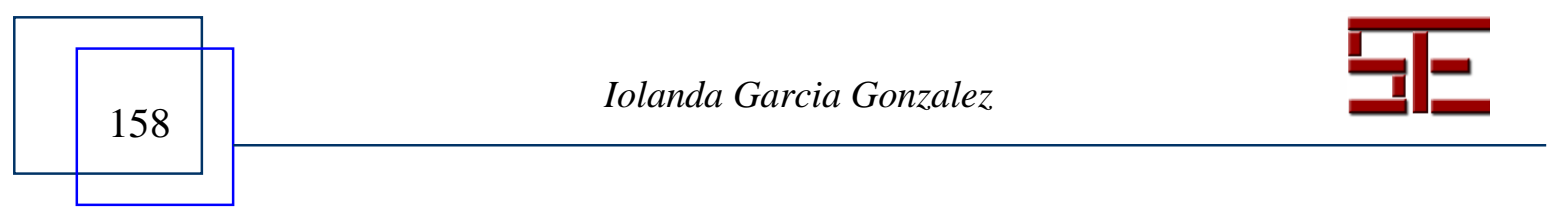




\section{A) ESTRUCTURA Y NAVEGABILIDAD}

$>$ Opciones de organización y visualización de las aportaciones: Los usuarios pueden ordenar y visualizar los mensajes en función de varios criterios, de la forma que más se adecúe a sus preferencias (pendientes de lectura, orden cronológico).

$>$ Estructuración en temas: Pueden crearse tantos temas o subámbitos de discusión como se quiera dentro de un mismo foro, en base a criterios organizativos de los grupos de participantes, temáticos o de contenido, de gestión de recursos, etc.

$>$ Buscador: Mediante dos opciones de búsqueda (simple y avanzada), los usuarios pueden recuperar la información publicada en un foro específico o en varios, a través de palabras clave. El sistema permite, además, búsquedas acotadas a periodos de tiempo específicos.

$>$ Sistema de sindicación vía RSS: Con objeto de facilitar el seguimiento de la actividad, eKnowledge ofrece la posibilidad de subscribirse a todos los foros activos dentro del aula, o bien selectivamente a un foro o tema en concreto. También es posible subscribirse a la actividad de un usuario en concreto.

$>$ Exportación y descarga de contenidos en formato .pdf: Descarga selectiva de los mensajes por temas, en forma de documentos en formato .pdf, con la intención de facilitar la gestión del tiempo de trabajo on line y off line.

$>$ Gestión de ficheros adjuntos: Cada usuario puede acceder a su espacio personal desde donde gestionar los documentos adjuntos que ha aportado personalmente a los foros.

$>$ Gestión de borradores: Los mensajes editados se pueden guardar como borradores antes de ser publicados. De este modo, los usuarios disponen de un espacio donde guardar y gestionar sus aportaciones al foro antes de hacerlas visibles para el resto de usuarios.

$>$ Seguimiento de actividad: Los docentes pueden acceder a la información relativa a la participación de los estudiantes en los foros mediante el registro de actividad individual de cada estudiante y valorar de forma más ágil la cantidad y la calidad de sus intervenciones.

Tabla 2. Descripción de las principales funcionalidades de eknowledge (1/2)

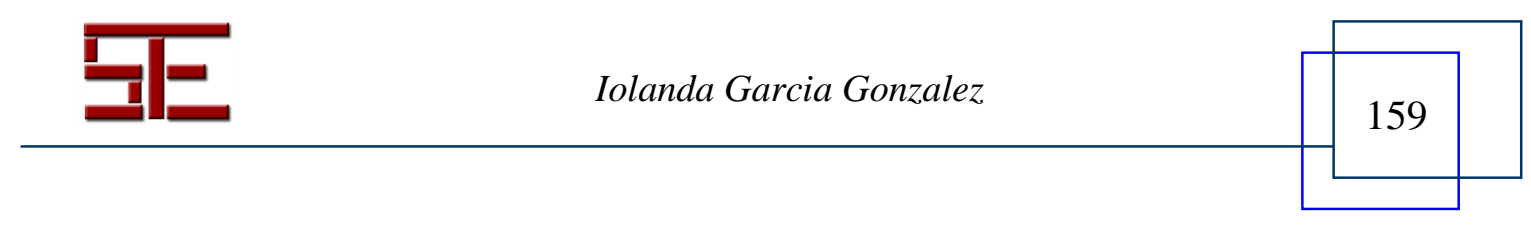




Teoría de la Educación
Educación y Cultura en la
$\begin{gathered}\text { VNiVERSiDAD } \\ \text { BSALAMANCA }\end{gathered}$

\section{B) APOYO CONSTRUCCIÓN CONJUNTA CONOCIMIENTO}

$>$ Aportaciones: Las opciones de edición de las aportaciones permiten incorporar una serie de elementos al cuerpo de mensaje (visuales y multimedia, palabras clave, etiquetas, referencias al contenido de otras aportaciones o espacios de participación, etc.), aparte de la posibilidad de adjuntar archivos en diferentes formatos, que favorecen la reflexión en torno al contenido, con el fin de caracterizarlo y a la vez profundizar en su comprensión y enriquecer la comunicación. Estos elementos facilitan también, por lo tanto, la identificación del contenido de las aportaciones sin necesidad de leerlas íntegramente. Las aportaciones se pueden guardar sin ser publicadas, de manera que es posible continuar su elaboración en otro momento y decidir cuando se hacen visibles al resto de participantes.

$>$ Valoración social de las aportaciones: Los usuarios pueden valorar cada mensaje enviado a los foros mediante la asignación de estrellas, en una escala de 1 a 5 . El sistema hace la media de las votaciones de los usuarios y muestra el resultado global. Situando el cursor sobre las estrellas se obtiene la información sobre el número de votos realizados y la media obtenida. Cada usuario puede votar una única vez y, si lo hace más de una vez, el valor que se registra es siempre el de la última votación.

$>$ Reedición de aportaciones: Una vez publicado un mensaje, puede editarse de nuevo y realizarse las modificaciones que se consideren necesarias. Después de una reedición, queda indicada dentro del cuerpo del mensaje la fecha de la última actualización y el nombre del autor.

$>$ Cita: Los usuarios pueden hacer aportaciones nuevas haciendo referencia directa a aportaciones anteriores mediante la opción de cita. Esta opción puede ser útil también para responder al mensaje de otro compañero de forma más directa y evidente. De este modo, el usuario puede escribir sus comentarios, conservando en el cuerpo de su nuevo mensaje, la aportación del original.

$>$ Referencia cruzada: Para potenciar la máxima interrelación de los contenidos aportados a los foros por diferentes usuarios, estos disponen de una funcionalidad específica que permite enlazar los nuevos mensajes con otros mensajes publicados anteriormente, tanto dentro del mismo tema y foro de discusión, como en otros temas. La herramienta de búsqueda permite identificar el o los mensajes que se quieren enlazar e incorporar un vínculo que haga posible la lectura de los mismos desde el mensaje original.

$>$ Etiquetas: A través de las etiquetas, los usuarios pueden identificar y clasificar con palabras clave el contenido de las aportaciones. Las etiquetas se vinculan a mensajes específicos, pero a la vez se acumulan a nivel de tema y de foro en forma de nubes de etiquetas, para identificar los contenidos generales de estos. Estas nubes permiten,

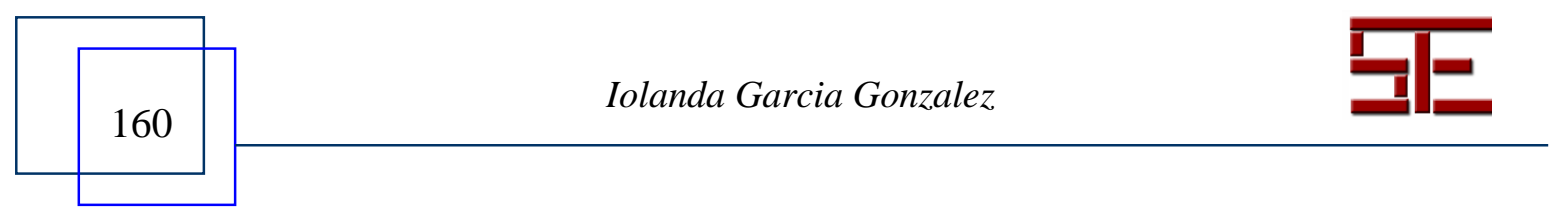




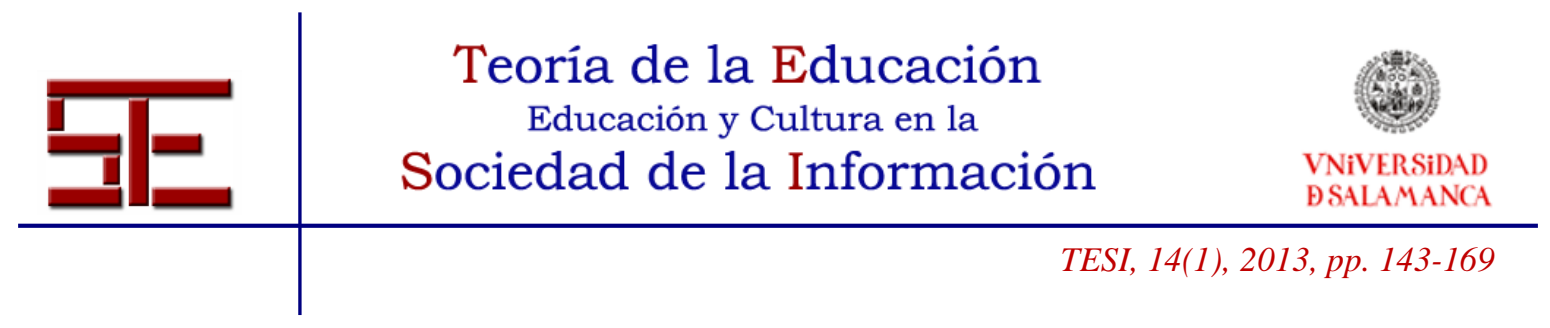

además, la navegación entre etiquetas y un acceso ágil al conjunto de aportaciones que han empleado una etiqueta específica. Del mismo modo que sucede con las folksonomias, el etiquetado de los contenidos de eKnowledge viene dado por la acción colectiva de sus usuarios.

$>$ Categorías: Los docentes pueden definir un conjunto de categorías que los estudiantes tendrán que asignar a cada uno de sus mensajes para expresar la función del contenido de aquella aportación. La finalidad de esta opción es favorecer la reflexión por parte de los estudiantes sobre sus propias aportaciones, durante la participación en los foros. También permiten estructurar las intervenciones del foro de acuerdo con unos determinados criterios. Por ejemplo, en un proceso de documentación y debate alrededor de un tema podrían ser útiles las categorías siguientes: "argumento a favor o en contra", "interrogante", "teoría", "información contrastada", "ejemplo", etc. A la vez, la categorización de las aportaciones proporciona al profesor información inmediata sobre el tipo de proceso que se está produciendo, le facilita la tarea de gestión de las aportaciones y le ayuda a identificar en qué momentos y de qué manera debe intervenir.

Tabla 2. Descripción de las principales funcionalidades de eknowledge (2/2)

Para desarrollar el primer prototipo de eKnowledge se decidió utilizar como plataforma base una herramienta de software libre y código abierto. Después de analizar distintas opciones se optó por la plataforma de foros phpBB. eKnowledge es, por lo tanto, una adaptación de la plataforma de foros phpBB, basada en el lenguaje de programación php. Las posibilidades de personalización, la versatilidad de las funcionalidades, así como la amplia comunidad internacional de apoyo y desarrollo con que cuenta esta plataforma, fueron algunos de los aspectos que motivaron la elección. Este proceso de adaptación implicó renunciar, al menos inicialmente, a la incorporación de algunas características de la maqueta inicial, bien por el hecho de requerir de una programación demasiado compleja, o bien para no disponer del tiempo necesario para hacer el desarrollo. Como contrapartida, no obstante, se añadieron otras funcionalidades no previstas, que ya incorporaba la plataforma phpBB. En la Tabla 2 se describen las principales funcionalidades que finalmente incorpora el primer prototipo de eKnowledge.

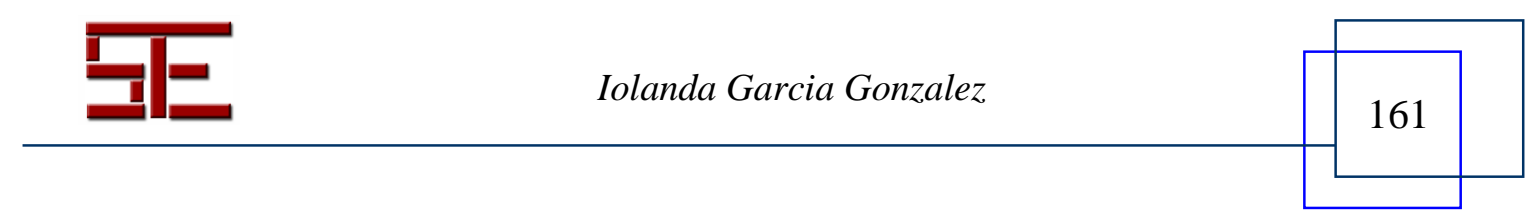




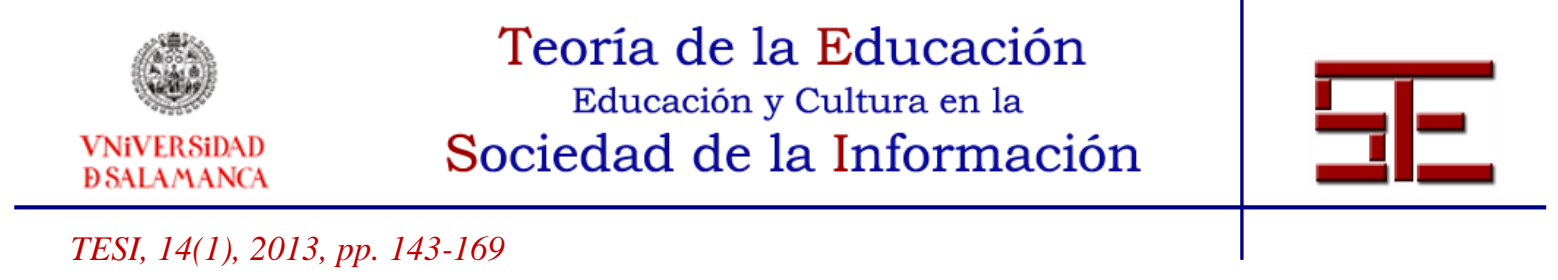

\section{5.- IMPLEMENTACIÓN Y ANÁLISIS DE PROCESOS DE CONSTRUCCIÓN CONJUNTA DE CONOCIMIENTO.}

Tal como comentábamos en las primeras páginas de este capítulo, la finalidad del proyecto, más allá de desarrollar una herramienta de foros sustituta del actual, es fomentar las prácticas de aprendizaje basadas en la comunicación y la colaboración. Éramos conscientes y hemos podido corroborar que lograr este objetivo implica algo más que poner al alcance de docentes y estudiantes una herramienta tecnológica, aunque se trate de una herramienta que incorpore funcionalidades para apoyar este tipo de procesos.

Una de las constataciones importantes a partir de los resultados de las encuestas realizadas ha sido el escaso uso que se ha hecho de las funcionalidades más complejas, es decir, aquellas más directamente concebidas para apoyar procesos reflexivos y argumentativos conjuntos entre los estudiantes. En gran medida, el motivo ha sido el desconocimiento de las funcionalidades en cuestión, pero sobre todo el hecho de no saber cómo sacar partido de las mismas.

Estas funcionalidades responden a usos que no se dan de manera espontánea, sino que responden a un modelo formativo determinado. A pesar de haber insistido en ello, en la mayoría de casos no se diseñaron actividades que requirieran la utilización de estas funcionalidades y, al no resultar necesarias, lógicamente no se utilizaron. Esto es aplicable, especialmente, a las categorías, las etiquetas y las referencias cruzadas.

Esto nos permite constatar la necesidad de profundizar en el uso de la herramienta en el marco de actividades de enseñanza-aprendizaje "prototípicas", es decir, vincular los usos de la herramienta con las finalidades de aprendizaje y el contexto didáctico de uso. Esta "puesta en práctica" de la herramienta en el marco de actividades de aprendizaje específicas puede ser útil tanto para valorar si proporciona los apoyos adecuados en el momento en que son necesarios, como para identificar, en consecuencia, dónde se requieren más "ayudas educativas", ajustadas a las necesidades de aprendizaje que presente cada situación particular.

No obstante, más allá incluso del conocimiento de la herramienta y de su adecuado encaje en el diseño de la actividad de aprendizaje, generar procesos de construcción colaborativa de conocimiento entre los estudiantes no es una tarea fácil. Tal como comentábamos en el inicio del artículo, resulta costoso conseguir que los estudiantes profundicen realmente en las ideas y se mantenga una cierta intensidad en la discusión, durante el tiempo

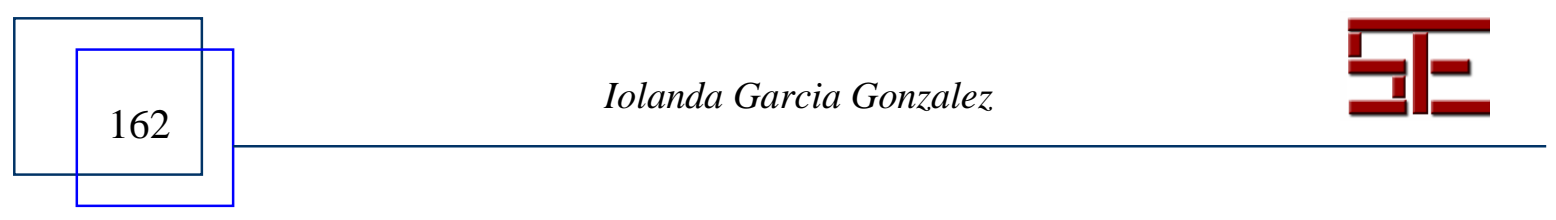




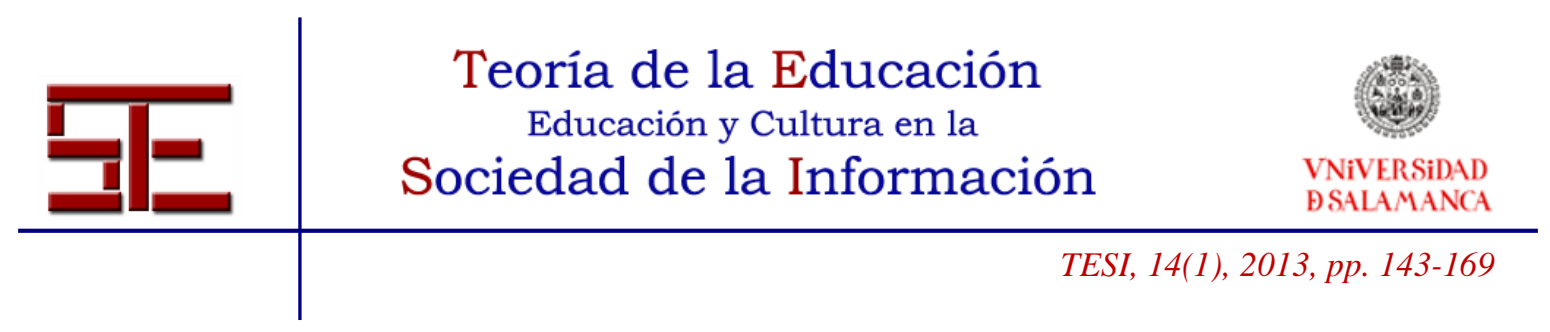

suficiente para que se produzca un excedente en términos de conocimiento (Marcelo y Perera, 2007; Wallace, 2003).

En esta línea, ofrecemos a continuación algunas pautas para la implementación de herramientas de comunicación asíncrona como apoyo de procesos de aprendizaje colaborativo o de construcción colaborativa de conocimiento (Andresen, 2009; Zhu, 2006):

a) Proporcionar el tiempo suficiente para la preparación y la generación de una discusión profunda y compleja. Algunas posibilidades son proponer una lectura de referencia, plantear una pregunta o preguntas que inciten la discusión o ejemplos en base a los cuales argumentar y posicionarse.

b) Hacer evidente la relación entre los temas y/o preguntas planteadas en la discusión, y los objetivos del curso, los recursos y materiales de aprendizaje de referencia. Las preguntas deben conducir la discusión a un terreno lo más concreto posible, partiendo de situaciones, fenómenos y acciones específicas, evitando así las vagas generalidades.

$\rightarrow$ El uso de la funcionalidad "Etiquetas" permite a los participantes identificar los conceptos clave de los temas tratados en las distintas aportaciones (y en el conjunto de la discusión), con el objetivo de hacer más visible y consciente el contenido fundamental de las mismas.

c) Transferir a los estudiantes la función de dinamización de la discusión, bien asignando roles abiertamente, o atribuyendo indirectamente este rol a aquellos estudiantes que participen más activamente. Mantenerse en un segundo plano como docente, interviniendo únicamente para reconducir o aclarar dudas y comentarios erróneos.

d) Estimular el uso de la síntesis de ideas, y la construcción a partir de las ideas de las aportaciones previas y las conexiones y referencias mutuas entre distintas aportaciones que pueden ser complementarias, contradictorias, de ampliación, etc.

$\rightarrow$ El uso de las funcionalidades "Referencia cruzada" y "Cita" es útil para recuperar el contenido de aportaciones previas, relacionar distintas aportaciones entre sí y hacer más evidente la conexión entre el contenido de unas y otras.

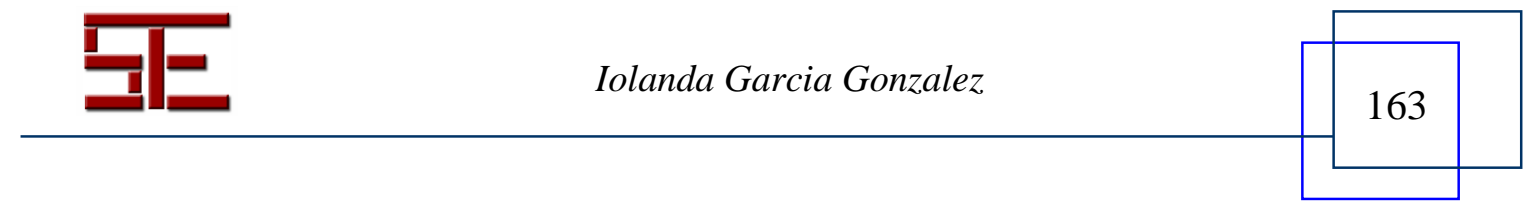




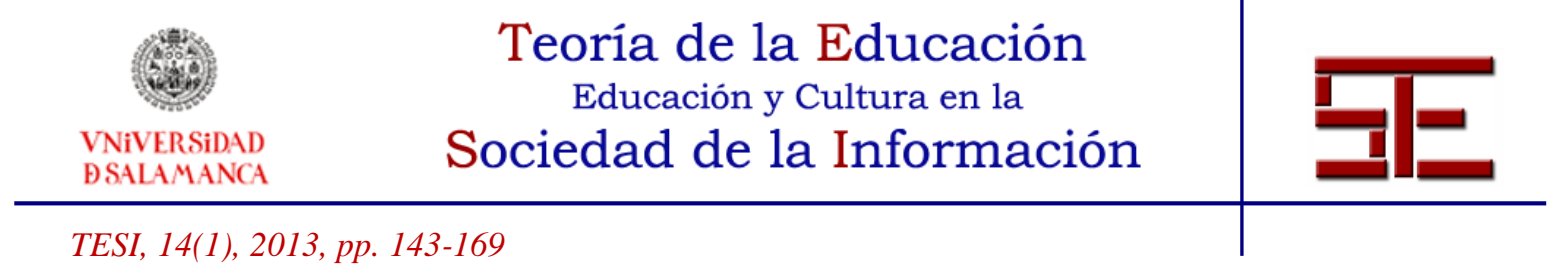

e) Estimular la argumentación y la fundamentación de las ideas planteadas (utilizando referencias teóricas si es necesario). Proporcionar mecanismos para dotar a la discusión de una estructura determinada en función del tipo de proceso que se pretenda generar.

$\rightarrow$ El uso de la funcionalidad "Categorías" permite clasificar las aportaciones en función de su finalidad y de acuerdo con unos criterios que pueden fijarse a priori. Esta categorización puede utilizarse como mecanismo para promover procesos cognitivos de alto nivel, al estimular la elaboración de aportaciones más complejas (p.e. algunas categorías que pueden utilizarse son: teoría, información fundamentada, hipótesis, argumento en contra o a favor, nueva idea, nuevo interrogante, síntesis, etc.).

f) Clarificar desde el inicio los criterios de evaluación que se utilizarán: proponer un número mínimo y/o máximo de aportaciones y establecer unos criterios claros en lo que respecta al tipo de contribución esperada (contenido, forma, nivel de profundidad, etc.). Otra posibilidad es involucrar a los estudiantes en la valoración de las aportaciones, proponiendo mecanismos para que estos evalúen la calidad o la utilidad de las aportaciones de sus pares, argumentando o no esta valoración.

$\rightarrow$ El uso de la herramienta de "Valoración" puede ser útil para generar procesos de valoración social entre los participantes.

Otro de los aspectos más problemáticos y complejos asociados a la cuestión que estamos tratando es el análisis de los procesos de construcción colaborativa de conocimiento, para su evaluación. Existen bastantes propuestas de modelos de análisis de distintas características. Algunas de estas propuestas parten del análisis del discurso que se desarrolla en las discusiones virtuales asíncronas, las cuales han sido objeto de síntesis, revisiones y adaptaciones (Marcelo y Perera, 2007; Gros y Silva, 2007; Gallego y Gutiérrez, 2011).

Uno de los primeros trabajos que aborda la calidad de las aportaciones realizadas en un foro es el de Henri (1992), quien plantea distintos niveles o dimensiones de análisis de la interacción: participativa o social, interactiva, cognitiva y metacognitiva.

Gunawardena, Lowe y Anderson (1997) identifican cinco fases en el proceso de discusión que representan un avance hacia niveles progresivos de construcción colaborativa de conocimiento: 1) comparar/compartir información; 2) descubrimiento y exploración de disonancias o inconsistencias; 3) negociación y co-construcción de conocimiento; 4)

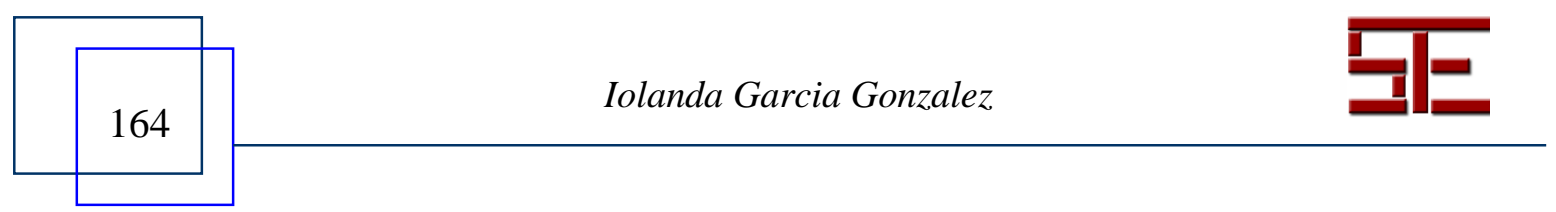




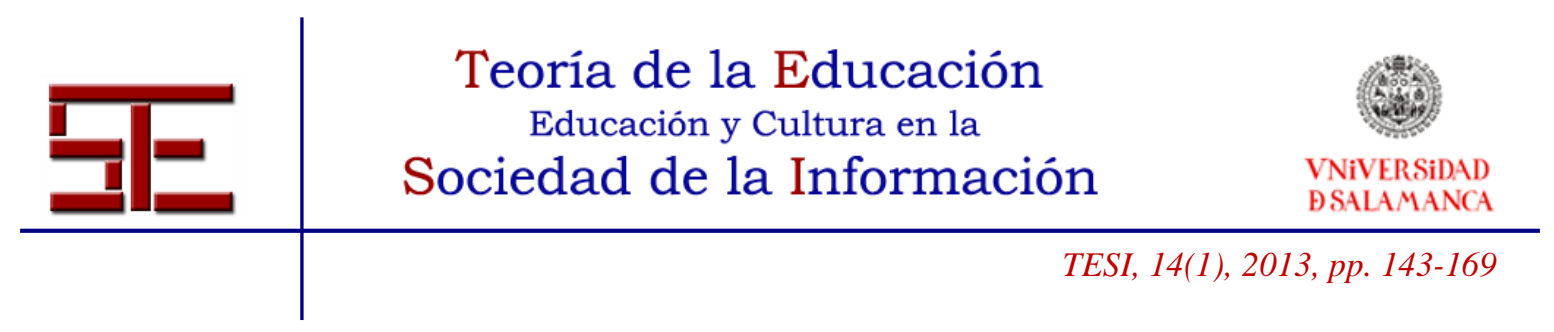

prueba y modificación de la síntesis propuesta; 5) acuerdo entre aportaciones y aplicación de nuevos significados.

Otro de los modelos más referenciados y utilizados en la actualidad es el propuesto por Rourke, Anderson, Garrison y Archer (2005). Desde una perspectiva más amplia, que abarca no una discusión en concreto, sino más bien el conjunto de aportaciones que tienen lugar en el seno de una comunidad de indagación virtual, estos autores proponen tres presencias o dimensiones fundamentales: social, didáctica o docente y cognitiva, con sus respectivas categorías e indicadores.

Otros autores se han centrado en analizar los niveles de complejidad cognitiva implícitos a las aportaciones (Gallego y Gutiérrez, 2011) o han desarrollado indicadores para observar el pensamiento crítico (Bullen, 1997). Scardamalia y Bereiter, (2002), a quien nos hemos referido anteriormente al presentar el programa Knowledge Forum, han propuesto también un sistema para la categorización de las aportaciones, basado en los principios de la argumentación o pensamiento progresivo. Las categorías se organizan en distintas series centradas en el proceso de construcción de conceptos, la formulación de opiniones y los principios de construcción de conocimiento, entre otros procesos relacionados siempre con la mejora y la elaboración progresiva de las ideas. Las categorías se asignan mediante "scaffolds" o andamiajes mediante la herramienta Knowledge Forum. De hecho, la herramienta presentada en este artículo incorpora la posibilidad de personalizar las categorías que incluir, por lo que puede adaptarse al sistema de codificación o categorización deseado, con el fin de apoyar distintos tipos de discusiones y facilitar el posterior análisis de las aportaciones.

Más recientemente se está explorando con la aplicación de nuevas técnicas de análisis con potencial para avanzar en este terreno. Es el caso de la minería de datos y concretamente del uso del enfoque de learning analytics, o del análisis de redes (social network analysis). En esta última aproximación, parámetros como la densidad, la centralidad o la centralización en las redes, por ejemplo, pueden ser indicadores de la cualidad de la interacción (Tirado, Aguaded y Hernando, 2012). Estas propuestas, todavía emergentes y poco generalizadas por su complejidad, representan un campo prometedor para el análisis y la evaluación de los procesos de construcción colaborativa de conocimiento en el marco del aprendizaje en red y a través de la web social.

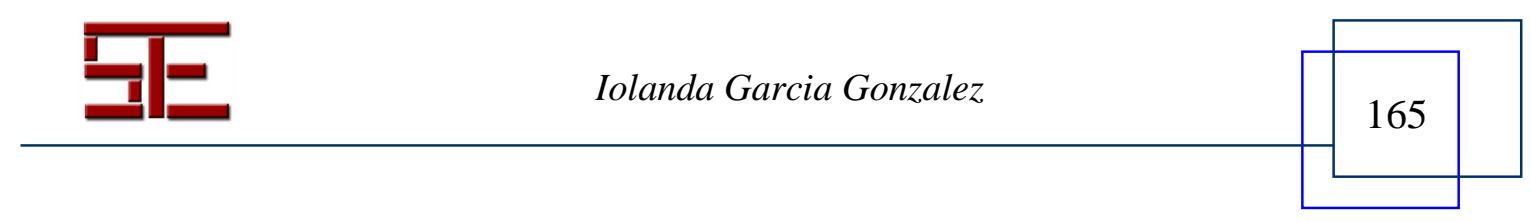




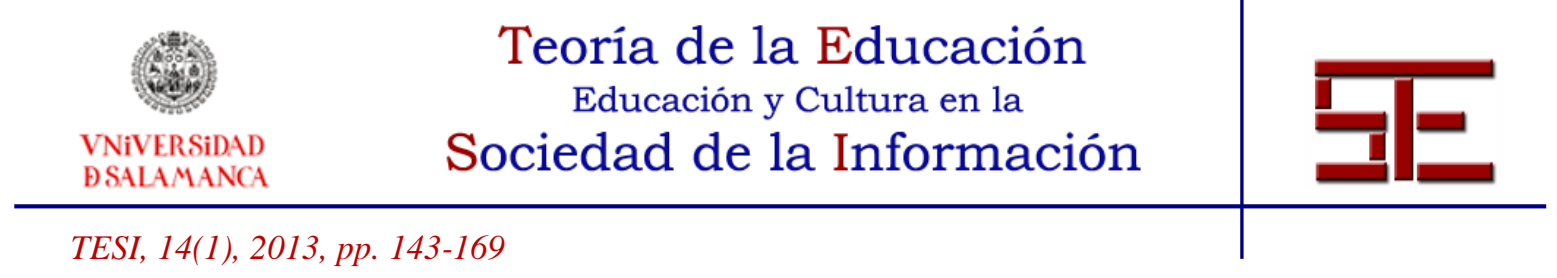

\section{6.- BIBLIOGRAFÍA.}

AA.VV. (2009). El modelo educativo de la UOC: evolución y perspectivas. Universitat Oberta de Catalunya <http://hdl.handle.net/10609/7262>.

Andresen, M. A. (2009). Asynchronous discussion forums: success factors, outcomes, assessments, and limitations. Educational Technology \& Society, 12 (1), 249-257.

Bereiter, C. y Scardamalia, M. (2003). Learning to work creatively with knowledge. En E. de Corte, L. Verschaffel, N. Entwistle y J. van Merrienboer (Eds.), Unravelling basic components and dimensions of powerful learning environments. EARLI Advances in Learning and Instruction Series.

Bullen, M. (1977). A case study of participation and critical thinking in university-level course deliverable by computer conferencing. University of British Columbia. Vancourver. https://circle.ubc.ca/handle/2429/6775.

Coll, C., Bustos, A. y Engel, E. (2011). Perfiles de participación y presencia docente distribuida en redes asíncronas de aprendizaje: La articulación del análisis estructural y de contenido. Revista de Educación, 354, 657-688.

Feliz, T. y Ricoy, M. C. (2008). El desafío tecnológico en el proceso de aprendizaje universitario. Los foros formativos. RELATEC: Revista Latinoamericana de Tecnologia $\begin{array}{llll}\text { Educativa, } & 7 & \text { (1), }\end{array}$ $<$ http://campusvirtual.unex.es/cala/editio/index.php?journal=relatec\&page=article\&op= view\&path[]=383>

Gallego, M. J. y Gutiérrez, E. (2011). Analizar la comunicación mediada por ordenador para la mejora de procesos de enseñanza-aprendizaje. Revista de Curriculum y Formación del Profesorado, 15, 1.

Gao, F. (2011). Designing a discussion environment to encourage connected and sustained online discussion. Journal of Educational Multimedia and Hypermedia, 20, (1), 43-59. http://pages.shanti.virginia.edu/cdg2011/files/2011/04/Discussion-Environmentto-Promote-Connected-and-Sustained-Online-Discussion.pdf.

Garrison, D. R., Anderson, T. \& Archer, W. (2000). Critical inquiry in a text-based environment: Computer conferencing in higher education. The Internet and Higher

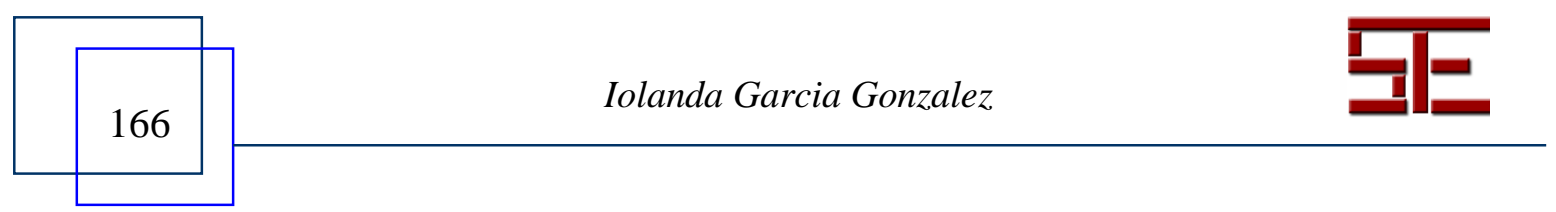




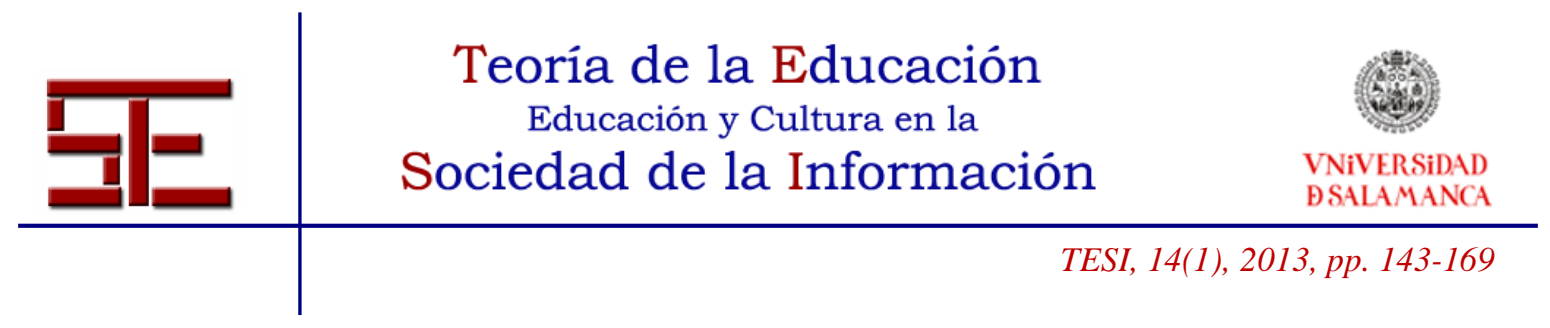

Education,
http://auspace.athabascau.ca:8080/dspace/bitstream/2149/739/1/critical_inquiry_in_a_te
xt.pdf

Gros, B. y Silva, J. (2006). El problema del análisis de las discusiones asíncronas en el aprendizaje colaborativo mediado. RED. Revista de Educación a Distancia, 16: http://www.um.es/ead/red/16/

Gunawardena, C. N., Lowe, C. A. y Anderson, T. (1997). Analysis of a global online debate and the development of an interaction analysis model for examining social construction of knowledge in computer conferencing. Journal of Educational Computing $\begin{array}{llll}\text { Research, } & 17 & \text { (4), 397-431. }\end{array}$ http://auspace.athabascau.ca:8080/dspace/bitstream/2149/772/1/ANALYSIS_OF_A_G LOBAL.pdf

Guzdial, M., y Turns, J. (2000). Effective discussion through a computer-mediated anchored forum. The Journal of the Learning Sciences, 9 (4), 437-469. <http://www.tandfonline.com/doi/abs/10.1207/S15327809JLS0904_3>

Hammond, M. (2005). A review of recent papers on online discussion in teaching and learning in higher education. Journal of Asynchronous Learning Networks, 9(3), 9-23. <http://www.aln.org/publications/jaln/v9n3/v9n3_hammons.asp>.

Henri, F. (1992). Computer conferencing and content analysis. En A. R. Kaye (Ed.), Collaborative Learning Through Computer Conferencing, (pp.117-136), Berlin: Springer-Verlag.

Hew, K. F., y Cheung, W. S. (2008). Attracting student participation in asynchronous online discussion: A case study of peer facilitation. Computers and Education, 51, 1111-1124. http://www.sciencedirect.com/science/article/pii/S0360131507001364.

Hewitt, J. (2005). Toward an understanding of how threads die in asynchronous computer conferences. The Journal of The Learning Sciences, 14 (4), 567-589. http://faculty.washington.edu/stkerr/Hewitt\%20Threads\%20die\%202005.pdf.

Kanuka, H. y Garrison, D. R. (2004). Cognitive Presence in Online Learning. Journal of Computing in Higher Education, 15 (2), 30-48.

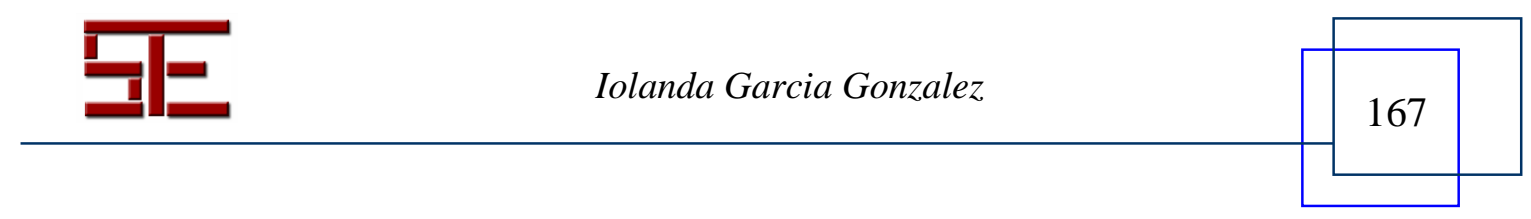




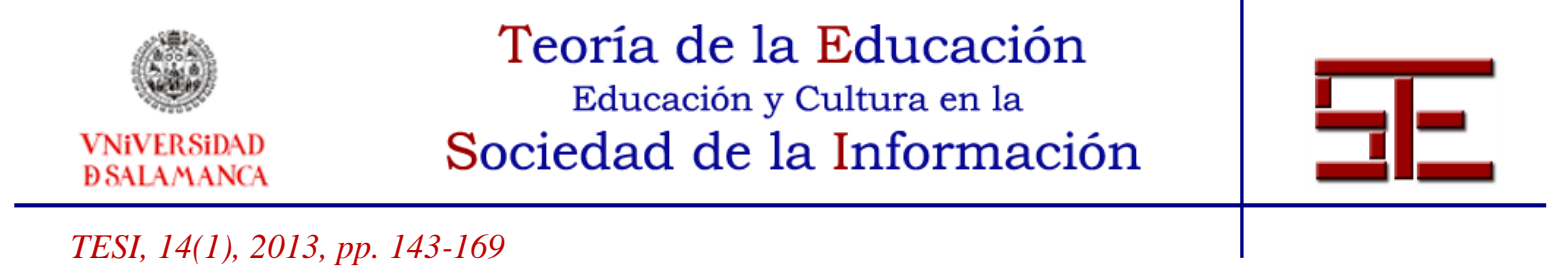

Kirschner, P., Strijbos, J. W., Kreijns, K. y Beers, P.J. (2004) Designing electronic collaborative learning environments. Educational Technology Research \& Development, 52 (3), 47-66. http://www.tlu.ee/ kpata/haridustehnoloogiaTLU/kirschner.pdf

Leinonen, T., Virtanen, O., Hakkarainen, K. y Kligyte, G. (2002). Collaborative Discovering of Key Ideas in Knowledge Building. Proceedings of the Computer Support for Collaborative Learning 2002 Conference. Boulder, Colorado, USA. <http://www2.uiah.fi/ tleinone/codi/two_page_codi_cscl.pdf >

Lipponen, L., Rahikainen, M., Lallimo, J., Hakkarainen, K. (2003). Patterns of participation and discourse in elementary students' computer-supported collaborative learning; Learning and Instruction, 13 (5), 487-509. <http://www.sciencedirect.com/science/article/pii/S0959475202000427>

Lomas, C., Burke, M. y Page, C.L. (2008). Collaboration tools. ELI Paper 2, August 2008. Educause Learning Initiative. http://net.educause.edu/ir/library/pdf/ELI3020.pdf

Marcelo, C. y Perera, V. H. (2007). Comunicación y aprendizaje electrónico: la interacción didáctica en los nuevos espacios virtuales de aprendizaje. Revista de Educación, 343, 381-429.

Rourke , L., Anderson, T., Garrison, D. R. \&Archer , W. (2005). Cuestiones metodológicas relativas al análisis de contenidos de las transcripciones de clases por ordenador. En Garrison, D.R y Anderson, T. (2005), El e-learning en el siglo XXI: Investigación y práctica. (pp.175- 202). Barcelona: Octaedro.

Scardamalia, M. y Bereiter, C. (2002). Knowledge building. En Encyclopedia of education, second edition. New York: Macmillan Reference, USA. $<$ http://ikit.org/fulltext/inpressKB.pdf>.

Stahl, G. (2006). Group cognition: Computer support for building collaborative knowledge. Cambridge, MA: MIT Press.

Thomas, M. J. W. (2002). Learning within incoherent structures: The space of online discussion forums. Journal of Computer Assisted Learning, 18, 351-366. http://onlinelibrary.wiley.com/doi/10.1046/j.0266-4909.2002.03800.x/pdf

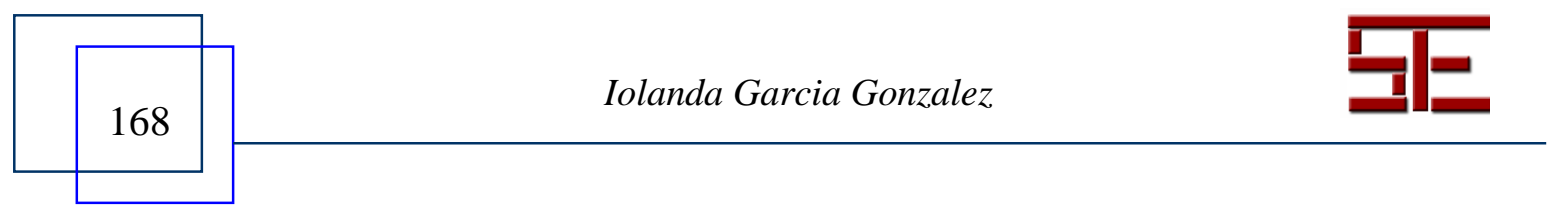




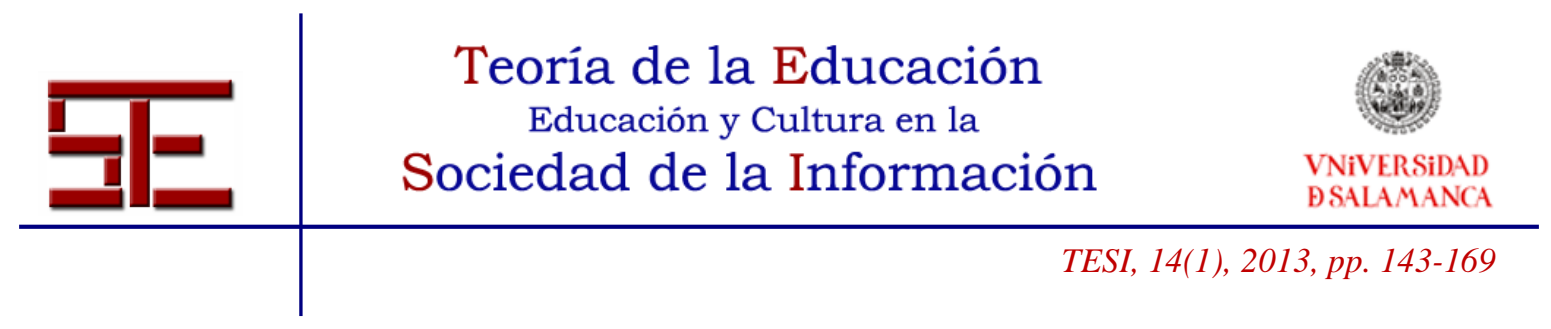

Tirado, R., Aguaded, I. y Hernando, A. (2012) Collaborative learning processes in an asynchronous environment: an analysis through discourse and social networks. Journal of Latin American Communication Research, 2 (1), 115-146.

Wallace, R. M. (2003). Online Learning in Higher Education: a review of research on interactions among teachers and students. Education, Communication \& Information, 3 (2), 241-280.

Zhu, E. (2006). Interaction and cognitive engagement: an analysis of four asynchronous online discussions. Instructional Science, 34(6), 451-480.

Para citar el presente artículo puede utilizar la siguiente referencia:

Garcia Gonzalez, I. (2013). Diseño funcional y propuesta de implementación de una herramienta de apoyo a la construcción colaborativa de conocimiento. Revista Teoría de la Educación: Educación y Cultura en la Sociedad de la Información. 14(1), 143-169 [Fecha de consulta: dd/mm/aaaa].

http://campus.usal.es/ revistas_trabajo/index.php/revistatesi/article/view/9447/9736

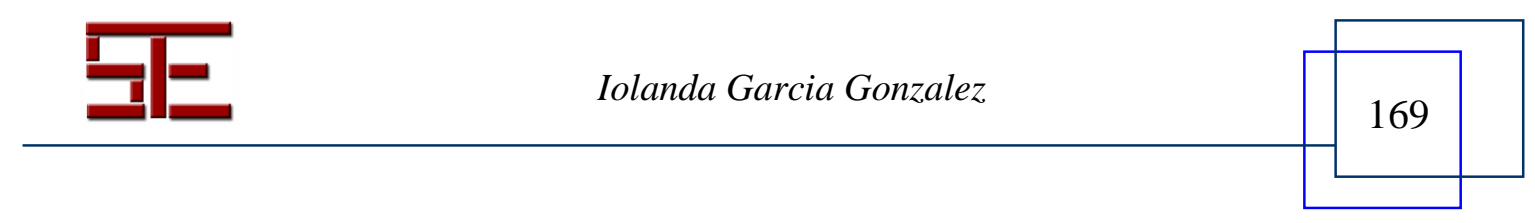

